\title{
The role of faceting in biaxially textured thin films: columnar morphology and abnormal tilting
}

\author{
Y. Huang, ${ }^{1}$ S. L. Masters ${ }^{2}$ S. P. Krumdieck, ${ }^{1}$ and C. M. Bishop ${ }^{1, \text { a) }}$ \\ 1) Department of Mechanical Engineering, University of Canterbury, Christchurch 8140, New Zealand \\ ${ }^{2)}$ School of Physical and Chemical Sciences, University of Canterbury, Christchurch 8140, New Zealand \\ (Dated: June 1, 2020)
}

The ground-breaking properties of biaxially textured thin films have attracted increasing attention to the characterization and growth theory of their crystal morphologies. In particular, multi-faceted columnar structures developed during oblique angle deposition (OAD) show abnormal tilt angles that have not been previously captured by existing models. Current theories for the formation of biaxially aligned columnar structures overlook the fact that the surface diffusion on individual facets can be finite. In this work, a continuum model incorporating finite adatom mobility, flux-dependent sticking coefficient and material-specific surface energies is employed to study the growth of a well-known MgO-OAD system. Experimentally observed morphologies are reproduced by simulating the shadowing growth of an array of preferentially oriented single crystals. We show that the abnormal tilting is elusive considering only the effects of faceting and shadowing. A proposed sticking coefficient in our model, determined by the component of adatom momentum parallel to the surface, is responsible for the development of abnormal tilting. The role of faceting is demonstrated by its effect on the resulting columnar morphologies. Using the proposed model, the characteristic morphology and tilting behavior of a $\mathrm{CaF}_{2}-\mathrm{OAD}$ system are also obtained which agree with experiments.

\section{INTRODUCTION}

In obliquely deposited thin films, symmetry-breaking columnar microstructures usually lead to special material properties, such as superconductivity ${ }^{1}$, photoelectric ${ }^{2}$, ferromagnetic ${ }^{3}$ and electrochemical ${ }^{4}$ properties. The performance of these properties is usually related to the columnar morphology developed during OAD. The origin of morphological development of columnar structures has been the subject of prolific and ongoing research in both areas of experimental and theoretical practice ${ }^{5,6}$. In general, the columnar morphology is sensitive to the degree of deposition asymmetry, characterized by a vapor incident angle $\alpha$. This asymmetry induces anistropic growth of the columnar structures, which results in tilting and texturing. In particular, the relationship between $\alpha$ and average tilt angle $\beta$, both measured with respect to the substrate normal, has been of great interest to the OAD theories. Among them, the empirical tangent law ${ }^{7}$

$$
2 \tan \beta=\tan \alpha
$$

and the cosine law ${ }^{8}$

$$
2 \sin (\alpha-\beta)=1-\cos \alpha
$$

agree with experiments for some OAD systems. While a large number of systems ${ }^{9}$ do not agree with these "universal" rules which do not account for differences in the materials deposited.

Based on a continuum model, Lichter et al. ${ }^{13}$ studied the tilting of columnar morphology in $d=1+1$ dimensions. The analytical solution of the tilt angle $\beta$ is found to be a function of $\alpha$ and a non-negative parameter $0<\Phi \leq 3.7$ determined by the adatom mobility:

$$
\tan \beta=\frac{2}{3} \tan \alpha /(1+\Phi \tan \alpha \sin \alpha)
$$

a) corresponding author

catherine.bishop@ canterbury.ac.nz

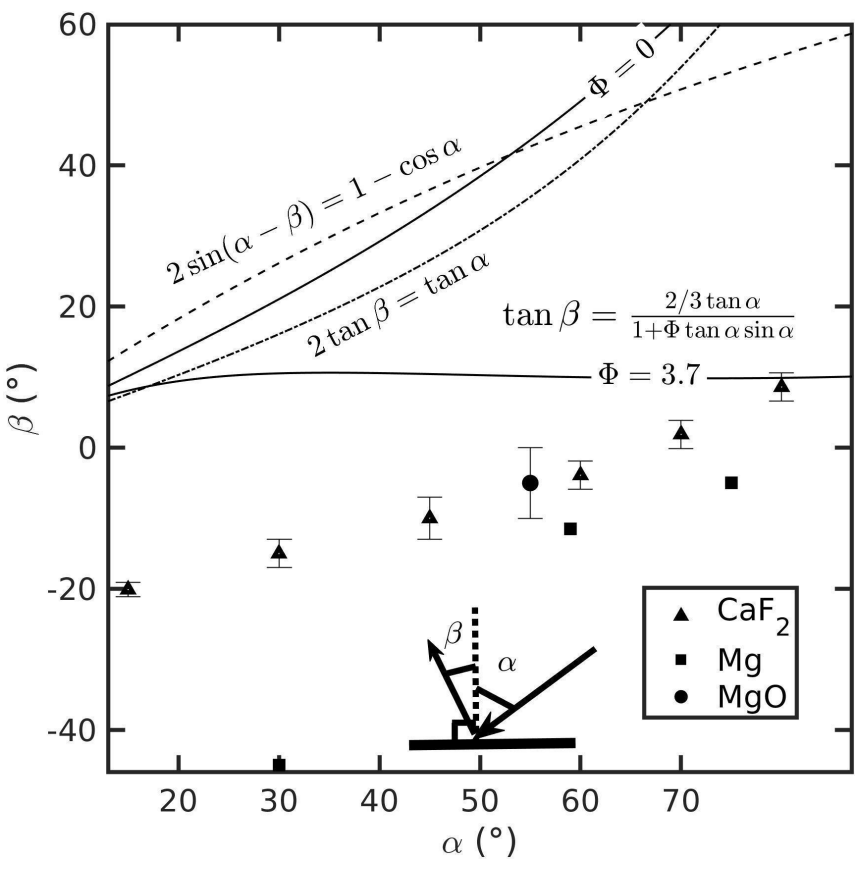

Fig. 1. Tilt angles $\beta$ for $\mathrm{CaF}_{2}{ }^{10}, \mathrm{Mg}^{11}$ and $\mathrm{MgO}^{12}$ compared to empirical laws and Lichter's formula. The inset depicts $\beta$ in relation to the flux angle $\alpha$, both measured with respect to the substrate normal, for the case $\beta<0$.

This is plotted in Fig. 1 for the limiting values of $\Phi$, along with the empirical laws. Eqn. 3 was successfully used to explain the tilting behaviours of some materials ${ }^{13}$. However, this approach employs small slope approximation and assumes large intercolumnar spacing. Thus, it neglects the overhang topology and shadowing effects altogether. These are important features to OAD systems, as far as tilting and columnar morphology are concerned. Keblinski et al. ${ }^{14}$ proposed a continuum model which admits overhanging configurations that are expected 
to arise from shadowing growth. The numerical solutions of their equations admit rich, non-linear morphologies of realistic columnar structures. Nevertheless, experimentally observed features, such as faceting due to anisotropic interfacial energy, are not considered in this model. Paritosh et al. ${ }^{15}$ used front tracking simulations to investigate the effect of shadowing on columnar morphology. $\beta$ vs $\alpha$ relations were numerically simulated. They are found to be dependent on the material symmetry, crystal orientation and the sticking coefficient. Shadowing is concluded to be responsible for the $\beta$ vs $\alpha$ relations. Although facets are featured in the geometric description of columnar structures, their model does not incorporate surface diffusion.

Despite the success in the above-mentioned models, there remain a number of biaxially textured thin films that produce $\beta$ angles deviating substantially from any of the existing model predictions. Nanostructured $\mathrm{MgO}$ thin films ${ }^{1,12,16,17}, \mathrm{CaF}_{2}$ nanorods $^{10,18}, \mathrm{Mg}$ nanoblades ${ }^{11,19}$, AlN columns ${ }^{20}$ and Mo-Al nanofins ${ }^{21}$ are examples of systems exhibiting abnormal tilt angles. The columns developed in these thin films are wellaligned single crystals tilted at angles that deviate significantly from existing predictions. Particularly, these thin films exhibit negative column tilt angles $(\beta<0)$, while existing models predict only $\beta \geq 0$. Instead of leaning towards the vapor flux, some columnar structures observed in these thin films are tilted away from the in-flux direction. Fig. 1 shows measured tilt angles for $\mathrm{CaF}_{2}{ }^{10}, \mathrm{Mg}^{11}$ and $\mathrm{MgO}^{12}$ (For some $\mathrm{MgO}$ systems ${ }^{16,17}$ negative tilting angles are also observed for $\mathrm{MgO}$ but $\beta$ values not reported). A new interpretation is needed for the origin of this deviation.

It has been speculated that the abnormal columnar microstructure may be related to atomistic adsorption energies ${ }^{10}$, which result in an anisotropic sticking coefficient as a function of surface crystallography. Others have suggested that the abnormal behaviour is due to the directional diffusion following the impingement of adatoms ${ }^{22}$. Monte Carlo simulations show that column-like structures can tilt away from the flux considering the directional diffusion of the adatoms in certain crystallographic directions ${ }^{17}$. Based on their suggestions, the nature of $\beta$ vs $\alpha$ relation should depend on the material being deposited. To date, there is no model that describes the detailed multi-faceted morphology and predicts the experimentally observed abnormal tilting of columnar structures.

It is widely recognized that the columnar microstructure in OAD films is due to non-local shadowing effects ${ }^{23,24}$. In addition, faceting due to the anisotropic surface energy is thought to be responsible for the multi-faceted morphologies commonly observed $^{10,12,19}$. In this work, we employ numerical simulations to investigate the role of faceting in determining the morphology of biaxially textured materials. The model is predicated upon a shadowing growth model proposed by Keblinski et al. ${ }^{14}$, which provides a good starting point for further studies incorporating the effects of faceting and sticking coefficient. Because the detailed morphology depends on the specific material being deposited, a well-known MgO-OAD system is chosen as an example for demonstrating the predictions of the model. In $\mathrm{Sec}$. II, we show that by faceting alone, the shadowing growth of $\mathrm{MgO}$ results in normal tilting. We then simplify and extend the model to include new physics for the incorporation of adatoms. The extended model not only accounts explicitly for the shadowing and material-specific surface energies, but also a proposed anisotropic sticking coefficient related to the component of adatom momentum parallel to the surface. By using the extended model, we show that abnormal tilt angles can be obtained. The multi-faceted, roof-tile morphology characteristic of a MgO-OAD system is reproduced by means of numerical simulation. In Sec. III, analysis of abnormal tilting, column bundling, terminating facets, growth exponent and void fraction are presented for $\mathrm{MgO}$. Lastly, the characteristic morphology and tilting of $\mathrm{CaF}_{2}$ columnar structures are simulated to confirm the possibility of applying the model to other material systems.

\section{SIMULATION METHODS}

\section{A. Shadowing growth}

Existing theories of the formation of biaxially textured thin films assume infinite surface diffusion of adatoms on a single crystal $^{25}$. A consequence of the unlimited surface diffusion is that the morphology of individual crystals is governed by the equilibrium shape and the film's microstructure is a result of grain boundary impingement. However, experimental observations show multi-faceted, nano-structured morphologies of isolated columnar structures that are high in porosity, rather than densely packed, fully faceted morphology attributed to infinite surface diffusion. It is therefore natural to presume a finite surface diffusion that allows the mobile adatoms to diffuse away, upon sticking, at a limited rate.

Continuum models capturing diffusional processes usually feature a coefficient of adatom mobility, which accounts for the effect of finite surface diffusion. For example, Keblinski et al. ${ }^{14}$ proposed a continuum model for the shadowing growth of a diffuse interface. In the deterministic part of this model, Eqn. 4 and Eqn. 5, $\phi(\boldsymbol{r}, t)$ and $g(\boldsymbol{r}, t)$ are conserved order parameters that take on equilibrium values $\phi=1, g=0$ in the solid and $\phi=-1, g=1$ in the vapor. $\phi$ and $g$, corresponding to the phasefield and vapor field respectively, are used to keep track of the material deposited and the vapor phase. The material interface is defined by the $\phi=0$ iso-surface, and the surface diffusion is modeled by the gradient flow of an energy functional $F . M$ is the coefficient that sets the strength of the adatom mobility, $\boldsymbol{J}$ is the flux that specifies the direction by which $g$ is advected. The shadowing effect is incorporated through the coupling of the two equations, with source and sink terms operative only in the interfacial region characterized by $(\nabla \phi)^{2}>0$. Coefficient $B$ is a sufficiently large number arbitrarily chosen to ensure efficient consumption of the $g$ field in this interfacial region, where the interface grows at the expense of the $g$ field.

$$
\begin{aligned}
& \frac{\partial \phi}{\partial t}=M \nabla^{2} \frac{\delta F}{\delta \phi}+B(\nabla \phi)^{2} g \\
& \frac{\partial g}{\partial t}=-J \nabla g-B(\nabla \phi)^{2} g
\end{aligned}
$$

In the implementation of this model in $d=2+1$ dimensions, 


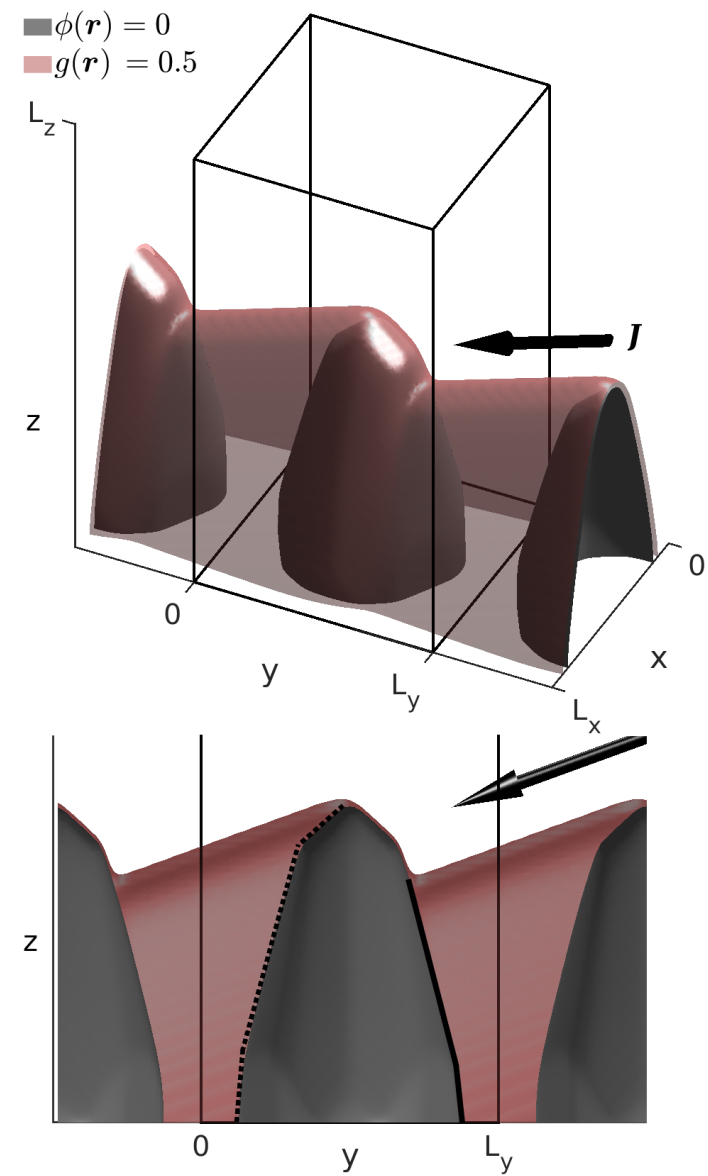

Fig. 2. Visualization of $\boldsymbol{J}$ and the iso-surfaces $\phi=0$ and $g=0.5$. The side view shows the solid line on the right which highlights the effect of global shadowing, and the dotted line delineated on the left side wall of the central column which marks the region of self-shadowing.

position vector $\boldsymbol{r}=(x, y, z)$ has three components and the simulation cell has dimensions $L_{x}, L_{y}$ and $L_{z}$. The shadowing growth of isolated single crystals is simulated by numerically solving the time evolution of $\phi$ and $g$ on a $256 \times 256 \times 512$ LegendreGauss-Lobato (LGL) grid, using an energy stable scheme ${ }^{26}$ and spectral-Galerkin method ${ }^{27}$. Periodic boundary conditions along $y$ are applied for $g$ to enable inter-columnar shadowing, whereas homogeneous Neumann boundary conditions are employed for the $\phi$ field. The inflow boundary condition at $z=L_{z}$ for the $g$ field is set to $g=1$, i.e. to maintain a constant supply of vapor. In Fig. 2, $\boldsymbol{J}$ and the material interface $\phi=0$ are visualized. An iso-surface of the vapor field $g=0.5$ is chosen for the visualization of three-dimensional shadowing effects. On the side view in Fig. 2, the shadowing on the central column and equivalently its periodic images in $y$ takes place in both side walls, with one overshadowed by itself (dotted line) and one facing the in-flux direction (solid line). The two should be distinguished, as the former is caused by the self-shadowing of the column and the latter is induced by the effect of global shadowing. We revisit this point in Sec. III.

\section{B. Material-specific faceting}

The multi-faceted crystal morphology of the biaxially textured columnar structures with abnormal tilt angles ${ }^{10,12,18-21,28}$ is widely considered to be associated with the interplay between geometric shadowing due to preferential deposition and the thermodynamics of faceting. Specific materials are considered in this work. Biaxially textured insulating films, such as $\mathrm{MgO}$ and $\mathrm{CaF}_{2}$, are technologically important semiconductor films exhibiting the above-mentioned features. The biaxially aligned $\mathrm{MgO}$ columnar structures with small angle grain boundary, for example, have applications such as buffer layers for growing superconductor films ${ }^{29}$. The good quality of the biaxial alignment allows the coated superconductor to achieve high critical current $^{1}$. The so-called "roof-tile" surface facets and multifaceted columnar morphology have been observed, regardless of the choices of substrate and fabrication technique ${ }^{12,16,28}$.

The surface free energy of many crystals is anisotropic and non-convex, which renders the gradient flow of energy $F$ in Eqn. 4 backward parabolic. To this end, we adopt a regularized energy functional ${ }^{30}$, Eqn. 6, defined over domain $\Omega$. This form of $F$ has been previously used for highly anisotropic film growth ${ }^{31-33}$.

$$
F[\phi]=\int_{\Omega} \frac{\gamma(\boldsymbol{n})}{\epsilon}\left(f(\phi)+\frac{\epsilon^{2}}{2}|\nabla \phi|^{2}\right)+\frac{\tilde{\epsilon}}{2 \epsilon^{3}}\left(f^{\prime}(\phi)-\epsilon^{2} \nabla^{2} \phi\right)^{2} d \Omega
$$

where $f=\frac{\phi^{4}}{4}-\frac{1}{2} \phi^{2}$ is the free energy density, $\epsilon$ the gradient energy coefficient, $\tilde{\epsilon}$ a regularization coefficient which sets the length scale of the corners and edges where facets meet and $\boldsymbol{n}(\boldsymbol{r})=-\nabla \phi /|\nabla \phi|$ is the outward pointing, unit normal to $\phi$ level set. Small numbers $\epsilon=0.02$ and $\tilde{\epsilon}=0.001$ are implemented in order to approximate a sharp interface.

The surface free energy density $\gamma(\boldsymbol{n})$ in Eqn. 6 for $\mathrm{MgO}$ is constructed using a method ${ }^{34}$ that has been adapted to suit the free energy formulation Eqn. 6 . We use ab initio-derived surface energy values for three important $\mathrm{MgO}$ surfaces ${ }^{35}$, $\gamma_{\{100\}}=0.93 \mathrm{Jm}^{-2}, \gamma_{\{110\}}=2.25 \mathrm{Jm}^{-2}$ and $\gamma_{\{111\}}=2.21 \mathrm{Jm}^{-2}$. The dimensionless surface energy density can be constructed by the formula:

$$
\gamma(\boldsymbol{n})=1-\sum_{i=1}^{26} a_{i}\left(\boldsymbol{m}_{i} \cdot \boldsymbol{n}\right)^{\omega_{i}} \Theta\left(\boldsymbol{m}_{i} \cdot \boldsymbol{n}\right)
$$

where the sum is over the 26 equivalent planes in the three families of surfaces, each with unit normal $\boldsymbol{m}_{i}$. The Heaviside function $\Theta\left(\boldsymbol{m}_{i} \cdot \boldsymbol{n}\right)$ has the effect of ensuring that $\boldsymbol{m}_{i}$ is distinguished from its opposite $-\boldsymbol{m}_{i}$ plane, which is not necessarily the crystallographically equivalent facet. $\omega_{i}$ and $a_{i}=1-\gamma_{i} / \gamma_{\{110\}}\left(1-a_{\{110\}}\right)$ are parameters that set the localization and the relative depth of the energy minima. Under this construction, the unspecified planes take on energy density equal to 1 far from minima and at orientations $\boldsymbol{n}=\boldsymbol{m}_{i}$ the energy density is equal to $1-a_{i}$. The $\gamma$-plot for $\mathrm{MgO}$ is shown in Fig. 3, juxtaposed with the equilibrium shape of the crystal reached by energy minimizing calculation such that the chemical potential $\delta F / \delta \phi$ is minimized. Following the details and 


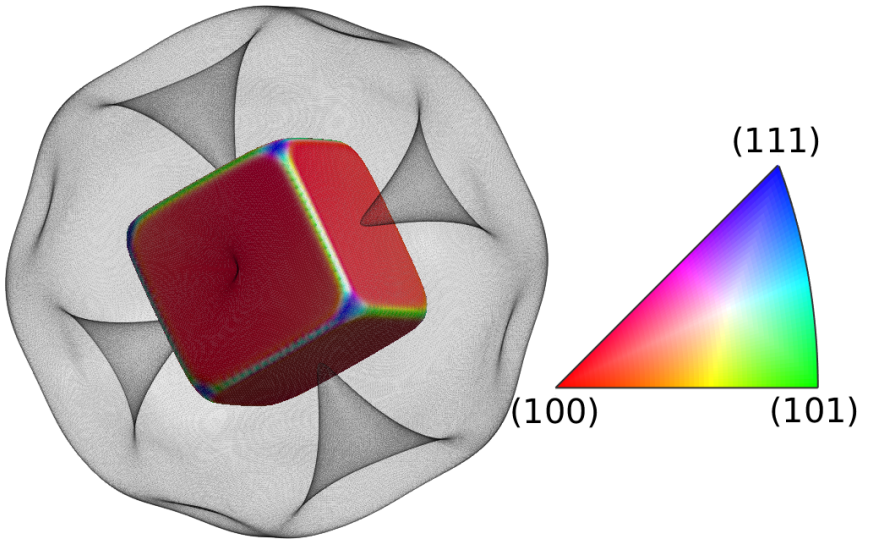

Fig. 3. Equilibrium shape of an isolated $\mathrm{MgO}$ crystal enclosed in the $\gamma$-plot.

applications of this $\gamma$-construction approach ${ }^{31-33}$, we choose $\omega=50$ and $a_{\{110\}}=0.1$ for all our simulations. The facets on the equilibrium shape are colored according to the stereographic triangle for cubic symmetry. The equilibrium cube shape is dominated by $\{100\}$ that possess the lowest surface energy and form a convex hull perpendicular to $\boldsymbol{n} \gamma(\boldsymbol{n})$. This is consistent with experimental observations and Wulff's theorem ${ }^{36}$.

\section{Columnar structures}

In this continuum approach, finite-size effects of the depositing species and atomistic processes with complications such as re-emissions are not included. Instead, the material interface and its dynamics are realized within the continuum model through the assumption of a diffuse interface. The deposition event is registered by the preferential exposure of the surface to the collimated vapor flux of adatoms owing to the $g$ field dynamics. By convention, the $z$ axis is selected as the substrate normal $\boldsymbol{n}_{s}$, and flux $\boldsymbol{J}$ is a unit vector, $\boldsymbol{J}=[0,-\sin (\alpha),-\cos (\alpha)]$, forming a deposition angle $\alpha$ with respect to $\boldsymbol{n}_{s}$. Subsequently, a crystal coordinate system $x^{\prime}, y^{\prime}, z^{\prime}$ is defined, so that these

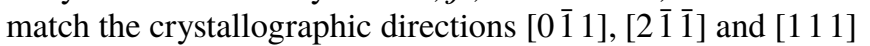
of $\mathrm{MgO}$. In many $\mathrm{MgO}-\mathrm{OAD}$ systems, the columnar structures develop a (111) out-of-plane texture and the texture axis [1 111$]$ tilts at an angle with respect to $\boldsymbol{n}_{s}$ for various $\alpha$ angles ${ }^{29,37,38}$. This can be characterized by the orientation angle $\beta^{\prime}$ of the lowest energy surface facing the flux. In the case of $\mathrm{MgO}$, this is the (100) facet angle, i.e. $\beta^{\prime}=\angle\left(\boldsymbol{n}_{s}, \boldsymbol{n}_{(100)}\right)$. $\beta^{\prime}$ should be distinguished from the column tilt angle $\beta$, which on the other hand specifies the physical inclination angle of the average growth direction of the columnar structures, independent of $\beta^{\prime}$. Although the preferred orientations $\beta^{\prime}$ for a limited number of materials ${ }^{39}$ can be fitted to a formula $\tan \beta^{\prime}=2 \tan \left(\alpha-\beta^{\prime}\right)$ given by van der Drift ${ }^{40}$, the nature of this orientation selection is complex. Biaxial texture typically improves with increasing film thickness. But the change in $\beta^{\prime}$ is found to slow down at later stages followed by only thickness growth ${ }^{19}$. Thus, it is reasonable to suggest that the well-aligned columnar morphologies are developed during the thickness growth after orientation

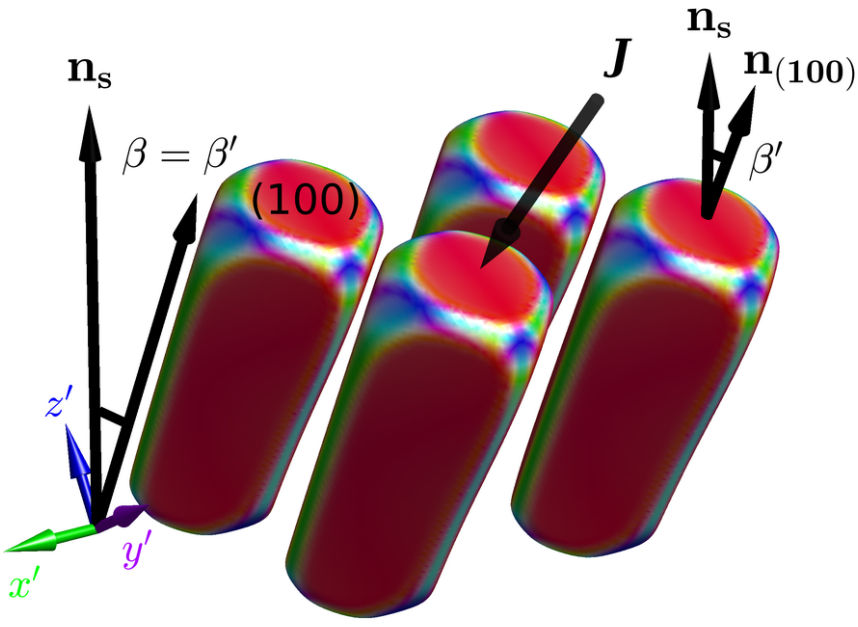

(a)

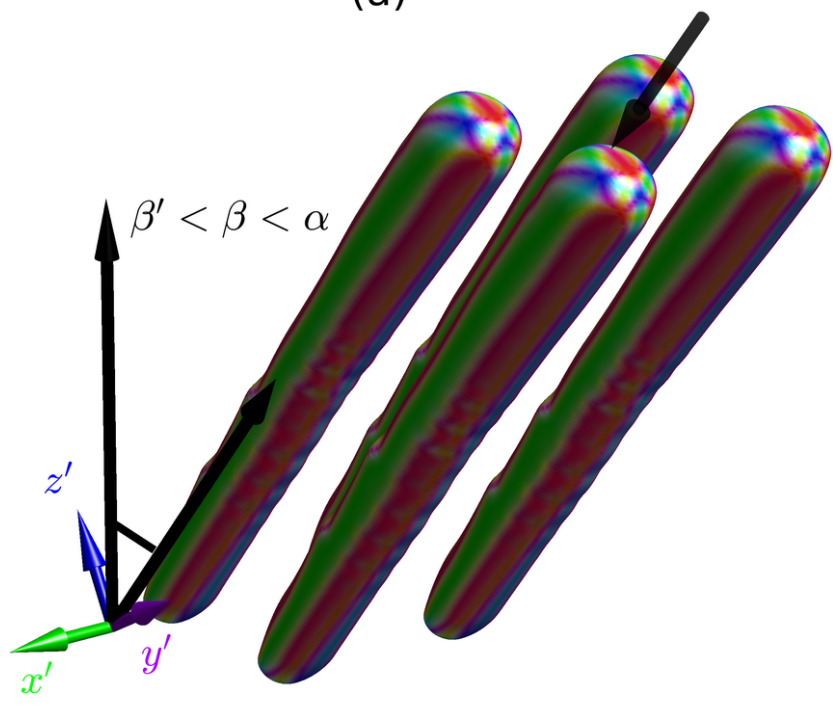

(b)

Fig. 4. Prototype $\mathrm{MgO}$ columnar structures grown under OAD with $\alpha=40^{\circ}$ and orientation of the seed set by $\beta^{\prime}=25^{\circ}$. The mobility of directional surface diffusion on facets are (a) $M=1$ (b) $M=0.01$.

selection with a fixed $\beta^{\prime}$. This is realized in our work by seeding the single crystals with preferred orientation according to the experimentally obtained $\beta^{\prime}$ values.

The relationships between $\alpha$ and $\beta^{\prime}$ reported in the literature differ only slightly for different $\mathrm{MgO}-\mathrm{OAD}$ systems, despite the differences in the choice of substrate and processing technique. In this work, we adopt the $\beta^{\prime}$ values measured by X-ray pole figures ${ }^{29}$. For a predefined biaxial texture, an array of hemispherical seeds with radius $r_{0}$ is oriented with a predefined (100) facet angle $\beta^{\prime}$ and with $x^{\prime}$ parallel to $x$. Each seed is separated by a distance $d_{0}=3 r_{0}$ from its neighboring seed in $x$ and $y$, and $L_{x}=L_{y}=2 d_{0}=1 / 2 L_{z}$ are conveniently chosen to accommodate four seeds per simulation cell. In order to isolate the effect of faceting driven by the adatom mobility, all parameters, except $M$, are fixed throughout the simulations. Therefore, we 


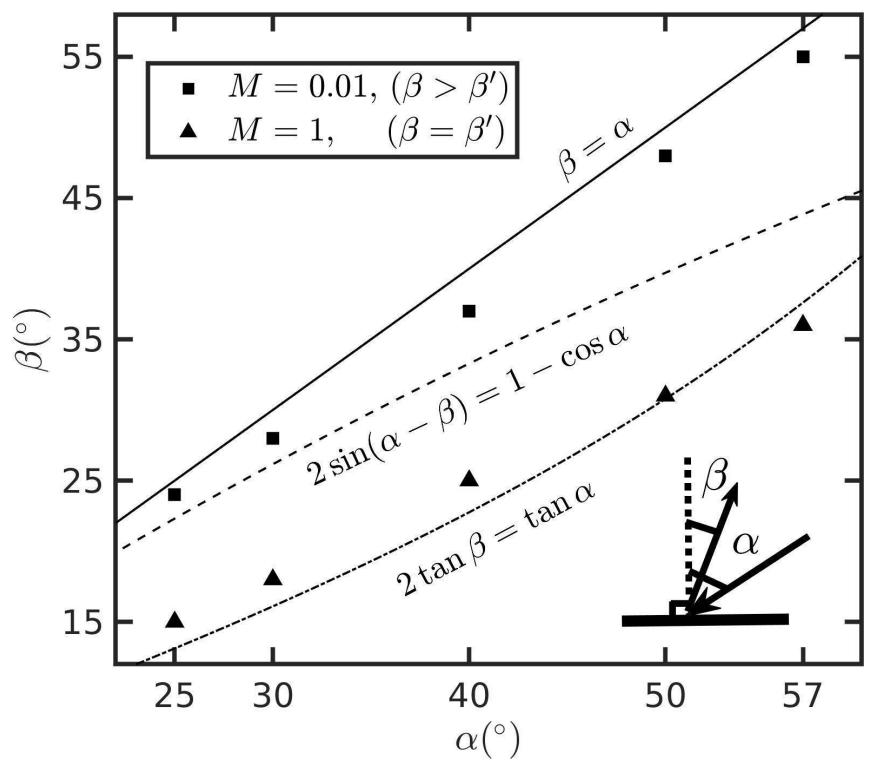

Fig. 5. Column tilt angle $\beta$ vs vapor incident angle $\alpha$ for $\mathrm{MgO}$ columnar structures according to Eqn. 4 and Eqn. 5.

Experimentally determined preferred orientations, specified by $\beta^{\prime}$ are taken from Xu et al. ${ }^{29}$ Also shown are the predictions of empirical laws.

consider here only the cases in which the columnar morphology is controlled by adatom mobility.

Two different $\mathrm{MgO}$ columnar structures developed under incident angle $\alpha=40^{\circ}$ are shown in Fig. 4. With $M=1$, Fig. 4 (a), the columnar structures develop $\{100\}$ facets and the average growth direction is parallel to $\boldsymbol{n}_{(100)}$, i.e. $\beta=\beta^{\prime}$. For $M=0.01$, Fig. 4 (b), $\beta>\beta^{\prime}$ and the columnar structures tilt more towards the flux with fewer facets formed. The area fraction of $\{100\}$ terminating planes in this case is smaller. The $\alpha$ dependence of tilt angles measured for the simulated columnar structures is shown in Fig. 5. The column tilt angle is controlled by adatom mobility. Simulation results for $M=1$ can be fitted to Lichter's formula with $\Phi=0.3$. $\beta$ decreases with increasing adatom mobility. For $M>10$ (not shown), the columns coarsen and approach the equilibrium shape. In this case, coalescence occurs without significant increase in thickness, until the columnar structures merge with each other. In actuality, small angle grain boundaries would form due to the small misorientations between columnar structures in biaxially textured thin films. Fully faceted columnar microstructures develop, for which the tilt angle $\beta=\beta^{\prime}$. We conclude that for the shadowing growth of preferentially oriented seeds according to Eqn. 4 and Eqn. 5, $\beta$ values obtained show normal tilting behavior and do not fall below $\beta=\beta^{\prime}$. In contrast, the multifaceted, biaxially textured thin films with abnormal tilt angle, $\beta<\beta^{\prime}$ are observed for $\mathrm{MgO}$.

\section{Realistic columnar morphologies}

For shadowing growth of practical interest, Eqn. 4 and Eqn. 5 can develop singularities that destabilize the numerical solution of $\phi$ and $g$. Keblinski included an artificial diffusion in Eqn. 5 to circumvent the problem ${ }^{14}$. But this is known to smear out the gradient of $g$ at some sharp edges and corners, and shadowing effects can be compromised. To allow for accurate tracking of the shadowing effects and to simplify the non-local dynamics, we propose here a local formulation for the rest of simulation results. To do this, the $g$ field can be approximated by a term of the form $S(\boldsymbol{r}) \boldsymbol{J} \cdot \boldsymbol{n}$ based on the simple fact that the value of $g$ advected to a point in the vicinity of the interface (flux divergence) is proportional to $\boldsymbol{J} \cdot \boldsymbol{n}$ with no shadowing. $S(\boldsymbol{r})$ is a probability, which assigns value $S=0$ if a grid point in the interfacial region is shadowed and $S=1$ otherwise. Ray tracing algorithm ${ }^{41}$ is used for the efficient computation of $S$ incorporating both self-shadowing and global shadowing effects. For a more general description independent of the dimension of simulation cell, the source term needs to be normalized by a factor $w(\boldsymbol{r})$ equal to the LGL quadrature weight, as $\int w(\boldsymbol{r}) d \boldsymbol{r}=L_{x} L_{y} L_{z}$. For the finite difference method used in the original numerical scheme of Keblinski's, $w$ is a constant. The same behavior shown in Fig. 5 can be reproduced by this approximation Eqn. 8, to the coupled Eqn. 4 and Eqn. 5.

$$
\frac{\partial \phi}{\partial t}=M \nabla^{2} \frac{\delta F}{\delta \phi}+B w(S \boldsymbol{J} \cdot \boldsymbol{n})(\nabla \phi)^{2}
$$

The shadowing growth according to Eqn. 8 does not give rise to $\beta$ value below $\beta^{\prime}$. It has been speculated that the momentum component of the incoming adatoms parallel to the surface is responsible for the variation in tilt angles ${ }^{22}$. Within our continuum model, the component of adatom momentum parallel to the interface is proportional to $|\boldsymbol{J} \times \boldsymbol{n}|$. Here we hypothesize that the quantity of adatom incorporation into the solid (as modeled by source term in Eqn. 8) is proportional to this momentum component. $|\boldsymbol{J} \times \boldsymbol{n}|$ can be understood here as a sticking coefficient. Being in this form, it varies between zero and one, with these limiting values obtained when $\boldsymbol{J}$ is parallel or perpendicular to $\boldsymbol{n}$, respectively.

For homoepitaxial systems, the sticking coefficient is usually considered a function of deposition parameters as well as surface crystallography ${ }^{42}$. In this case, it takes on different values for various surfaces regardless of their orientations with respect to the flux. However, for OAD films introduced in Sec. I varying from single component $(\mathrm{Mg})$ to multicomponent $(\mathrm{MgO})$ systems, complications arise due to the 3D morphology of columnar structures. The terminating surfaces of a columnar crystal are oriented differently with respect to the flux, depending on the surface normal $\boldsymbol{n}$ and $\boldsymbol{J}$. Further, the deposition conditions for OAD systems considered here also differ (e.g. vapor pressure, depositing species and substrate temperature). Experimentally, the deposition angle $\alpha$ determines the crystal morphology for OAD systems being modeled herein. It is therefore reasonable to include this additional term, in the source term in Eqn. 8. Our extended model takes the form: 


$$
\frac{\partial \phi}{\partial t}=M \nabla^{2} \frac{\delta F}{\delta \phi}+B w|\boldsymbol{J} \times \boldsymbol{n}|(S \boldsymbol{J} \cdot \boldsymbol{n})(\nabla \phi)^{2}
$$

Our treatment of the sticking coefficient takes account for the facet orientation and renders the adatom incorporation more efficient when the in-flux direction makes a larger angle with $\boldsymbol{n}$. This choice may be justified by the step flow growth mode which is commonly observed in multilayer thin film growth ${ }^{43,44}$. Under the step flow growth mode, the incorporation is more likely to occur when adatoms come to the surface at a larger angle with respect to $\boldsymbol{n}$. The adatoms are then captured by atomic steps and the layers grow laterally by the help of subsequent surface diffusion.

According to Eqn. 9, for a LGL grid point $\boldsymbol{r}$ on an interface associated with area proportional to $w(\boldsymbol{r})(\nabla \phi)^{2}$, the rate of growth is equal to the total flux reached at the interface $B(S \cos \gamma)$ multiplied by the sticking coefficient $\sin \gamma$, where $\gamma=\angle(\boldsymbol{J}, \boldsymbol{n})$ is the angle between the surface normal and the flux. Coefficient $B$ is fixed to $1 \times 10^{10}$ throughout the simulations along with time step $\delta t=1 \times 10^{-7}$, so that in practice for the range of $M$ employed in the simulations, $\phi$ field does not obtain far-from-equilibrium values. In addition, the source term $B$ is restricted to an interfacial region $|\phi|<0.95$ only. Without this restriction, the increase of $\phi$ is unbounded which deteriorates the numerical solution in the long runs ${ }^{14}$.

With the extended model, Eqn. 9, columnar structures having realistic morphologies can be derived. In particular, Vshaped columnar structures characteristic of OAD-MgO biaxially textured microstructure is obtained and shown Sec. III. We show that some experimentally observed morphological features, such as column bundling and abnormal tilting, can be accounted for by our extended shadowing growth model. Realistic multi-faceted morphologies and inter-columnar voids are also reproduced. The role of faceting is demonstrated by its effect on tilting and porosity, which is controlled by model parameter $M$ relating to the adatom mobility. The evaluation of the shadowing growth also predicted different power law behaviors before and after the column pinch-off. Lastly, by changing the material symmetry to a well-characterized $\mathrm{OAD}-\mathrm{CaF}_{2}$ system, we show that our model accounts for the experimentally observed tilting behaviors previously inexplicable.

\section{RESULTS}

\section{A. Growth mechanism and column bundling}

The resulting columnar morphology of the shadowing growth on two one-dimensional (1-D) arrays of seeds offset by distance $1 / 2 d_{0}$ in the direction facing the flux (in-flux direction) are shown in Fig. 6. This offset simulates an environment where a single column can be shadowed not only by its neighbor in the in-flux direction but also by neighbors in the $x^{\prime}$ direction. The surface morphology of the columnar structures has a $\{100\}$-type, roof-tile-shaped top facet (we denote them here by (100) to facilitate the discussion). The (100) top facets grow the fastest and the fastest vertical growth direction of (100)
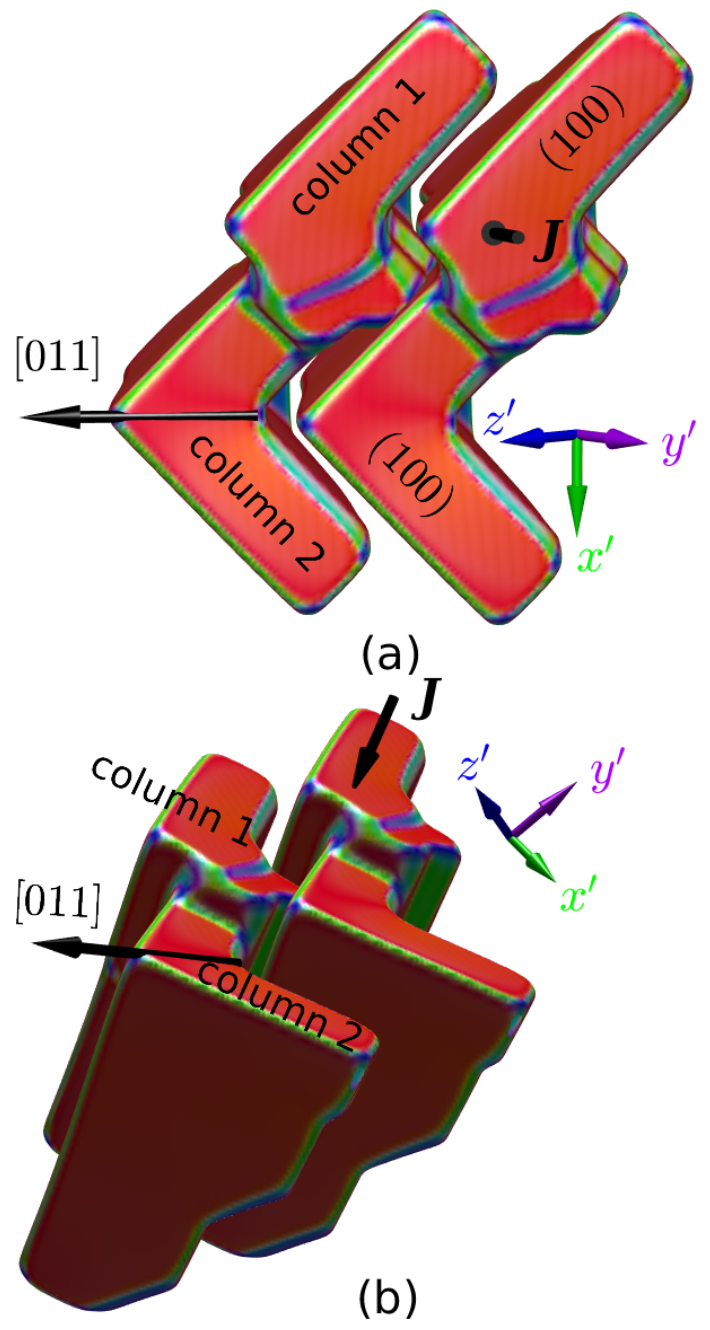

Fig. 6. (a) the top view and (b) the side view of column structures grown on two staggered 1-D arrays of preferentially oriented seeds with model parameters $M=0.5$. The top (100) facets, the fastest vertical growth direction of (100) and the flux $\boldsymbol{J}$ (corresponding to $\alpha=25^{\circ}$ ) are labeled.

appears to be [011]. Following the notation for biaxial relationships $^{6,18}$, we define the biaxial texture of the $\mathrm{MgO}$ columnar structures as $\{111\}\langle 110\rangle$. This means that the thin film has a (111) out-of-plane texture and an in-plane texture denoted by $\langle 110\rangle$.

Grown on top of preferentially oriented seeds, the columnar structures first undergo coarsening, accompanied by an increase in film density. At later stage the side wall facing the flux is completely overshadowed due to the inter-columnar, global shadowing. The self-shadowed side wall in contrast, is always shadowed and it hence receives no adatoms at all growth stages. As a result, the morphology on the global shadowing side is determined by the self-shadowed side wall of the neighboring column in the in-flux direction. The columns coarsen and eventually merge in the $x^{\prime}$ direction perpendicular to the flux. This causes a bias in the total flux reached at the top (100) facets at later stage, e.g. in Fig. 6 part of the (100) facet of 
column 2 is overshadowed. Column 2 has a smaller growth rate relative to column 1 accordingly. Columns aligned in the in-flux direction are not subject to this bias. As the growth furthers, column 1 receives more adatoms and is expected to eventually outgrow column 2 and this is evident on the side view Fig. 2 (b). As a result, columns grow competitively and line up in the $x^{\prime}$ direction due to this bias.

This phenomenon is known as column bundling, and it has been extensively observed in obliquely deposited, biaxially textured thin films. An example is shown by the microstructure of $\mathrm{Mg}$ nanoblades ${ }^{11}$, for which the blade-shaped columnar structures are well-aligned in the direction perpendicular to the flux. For $\mathrm{MgO}$ thin films, it has been reported that the fraction of column bundles is dependent on the quality of the biaxial alignment ${ }^{12}$. In our simulations, the biaxial alignment is perfect because of the predefined orientations. Different positioning of the seeds does not alter the observed competitive growth, as long as a bias in shadowed surface area persists. While Abelmann et al. ${ }^{22}$ attribute concerted bundling to selfshadowing, we show here that it is caused by global shadowing and the following competitive growth.

\section{B. Multi-faceted columnar morphology}

The shadowing growth of columnar structures in the presence of faceting and anisotropic sticking coefficient results in multi-faceted columnar morphologies. The terminating facets consist of predominantly $\{100\}$ facets, in agreement with the energetics. The fraction of $\{100\}$ facet area decreases with decreasing adatom mobility. This is demonstrated in Fig. 7 by two columnar structures developed during the thickness growth with different values of $M$. At earlier stage of growth, the top (100) facet adapts a fan-like shape with a $90^{\circ}$ fan angle. The columns have a mirror symmetry about $(0 \overline{1} 1)$. As the columnar structures coarsen, global shadowing hinders the adatom supply to the side wall and eventually part of the top facet. The morphology of individual columns is then determined by the positioning of neighboring columns. Particularly, asymmetry in morphology develops due to the bias in the direction $x^{\prime}$ perpendicular to the flux, as discussed in the previous section.

Columnar structures in Fig. 6 are perfectly aligned and the columns eventually merge and coalesce in the $x^{\prime}$ direction. This can be characterized by the growth of column width $W$, which stops when the grain boundary impingement occurs. X-ray pole figure analysis shows that there is a spread of in-plane and out-of-plane alignment in OAD deposited films ${ }^{29}$. Small angle grain boundaries are not included in our model. It is however reasonable to argue that the average growth direction of the columnar structures always converges during the thickness growth, regardless of the positioning of the columns and the small variation in orientations. We therefore consider a special neighborhood in which no columns in the $x^{\prime}$ direction are close to each other, as shown in Fig. 7. Inside of this neighborhood, $W$ continues to increase before reaching the edge of the simulation cell, and the mirror symmetry is retained. The average growth direction obtained can be used to inform the one in a more complex neighborhood. The initial seed separation is $d_{0}=3 r_{0}$

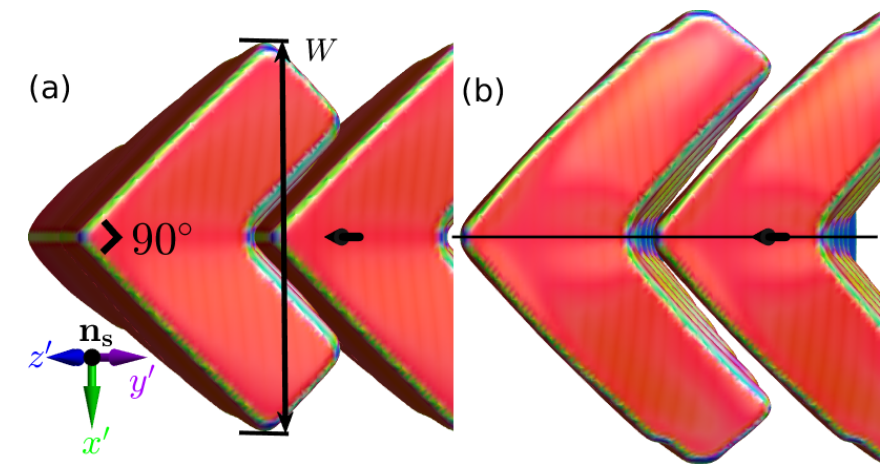

(c)

(d)

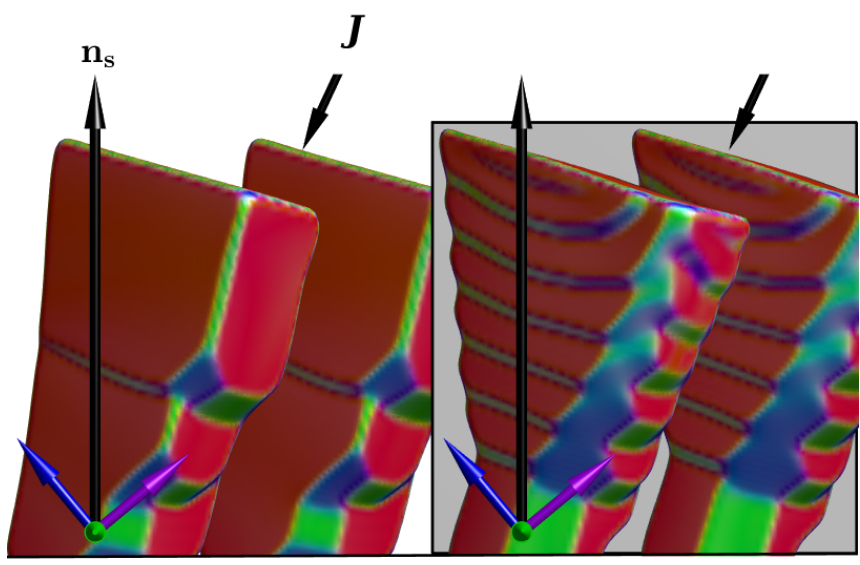

Fig. 7. Top view and side view of a 1-D array of multi-faceted columnar structures before they impinge onto the simulation cell with $M=1$ for (a) and (c); and $M=0.1$ for (b) and (d). Column width $W$ and flux $\boldsymbol{J}$ (corresponding to $\alpha=25^{\circ}$ ) are labeled. The (011) mirror plane for the columnar structures is highlighted in (b) and (d).

as before, but the substrate is seeded with 1-D array of single crystals lined up in the in-flux direction. The choice of $d_{0}$ does not affect the average growth direction, i.e. $\beta$ is independent of $d_{0}$.

As shown in Fig. 7 for columnar structures oriented at $\beta^{\prime}=15^{\circ}$ with deposition angle $\alpha=25^{\circ}$, the side view reveals the V-shaped, multi-faceted $\mathrm{MgO}$ columnar structures grown under shadowing effects. This $\mathrm{V}$-shaped overall morphology was previously thought to result from competitive growth ${ }^{12}$. Our simulation suggests that the origin of the V-shaped morphology is due to the inter-columnar, global shadowing during thickness growth. Moreover, the so-called "laminar structure" observed on the side walls of $\mathrm{MgO}$ columns was thought to originate from the repeated nucleation of $\{100\}$ facets $^{12}$. Our model suggests that the formation of alternating facets is due to the interplay between preferential growth and faceting. With smaller adatom mobility the terminating facets of the columnar structures take on energetically less favorable configurations. However, the origin and nature of the alternating facets on columnar structures due to non-equilibrium growth of the solidvapor interface can be complex. 


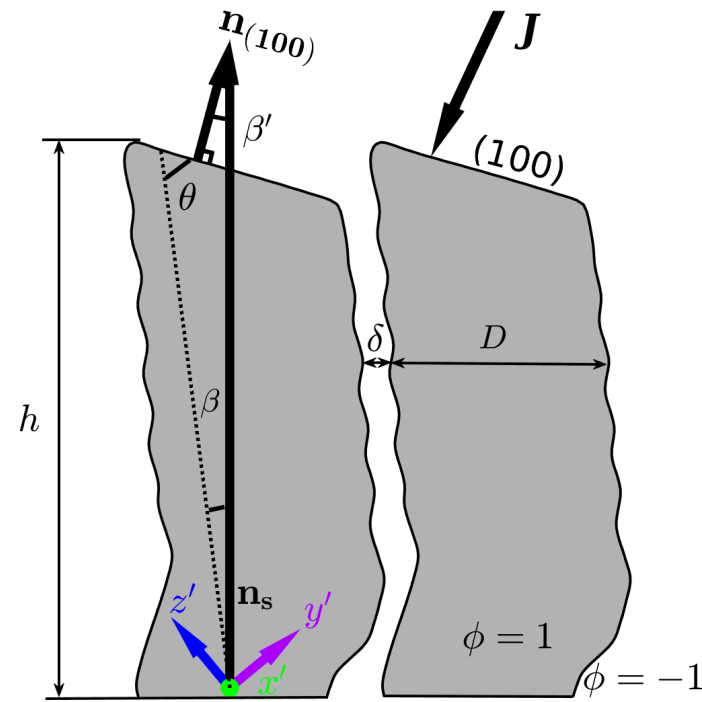

Fig. 8. A cross section along the $(0 \overline{1} 1)$ mirror plane of the $\mathrm{MgO}$ columnar structures in Fig. 7 (b) and (d). Morphological parameters $\beta, \theta, D$ and $\delta$ characteristic of the columnar structures are labeled for further analysis.

\section{Abnormal tilting}

In order to reliably estimate $\beta$, we first examine the V-shaped columnar morphology and define the average growth direction. The two side walls composed of sets of alternating surfaces (on side view in Fig. 7 (c) and (d)) grow in directions both deviating from the true average growth direction. In Fig. 8, the same columnar structures are cross sectioned through a mirror plane (Fig. 7 (b) and (d)). The cross section along the (01̄1) mirror plane reveals an average growth direction tilted away from the flux. The tilt angle corresponding to the deposition angle $\alpha=25^{\circ}$ in this example is $\beta=-8^{\circ}$. Our simulations show that larger adatom mobilities favor the growth of the columnar structures with tilt angle closer to $\beta=\beta^{\prime}$ and increase the $\{100\}$ area fraction. On the other hand, the kinetics of the shadowing growth induced by the preferential deposition and the anisotropy in the sticking coefficient renders the average growth direction away from $\beta=\beta^{\prime}$. Therefore, the observed tilting behavior is due to the combined effects of faceting thermodynamics and the kinetics.

The $\beta$ vs $\alpha$ plot for various $M$ is shown in Fig. 9. All results deviate significantly from the empirical laws plotted in Fig. 5. A dependence on the adatom mobility is evident. Larger $M$ values favor the coverage of $\{100\}$ facets on the side walls and result in $\beta$ values closer to $\beta^{\prime}$. Conversely, smaller $M$ values produce more alternating facets on the side walls and yield $\beta$ values that are further away from $\beta^{\prime}$. By comparison with the tilting behavior shown in Fig. 5, all tilt angles obtained are smaller than $\beta^{\prime}$. In some cases, negative column tilt angles were observed, i.e. $\beta<0$. Our work shows that this cannot be obtained by considering faceting and shadowing alone, Eqn. 4 and Eqn. 5. The abnormal tilting is attributed to the sticking coefficient determined by the momentum component of adatom parallel to $\mathrm{MgO}$-vapor interface. The inclusion of such anisotropic stick-

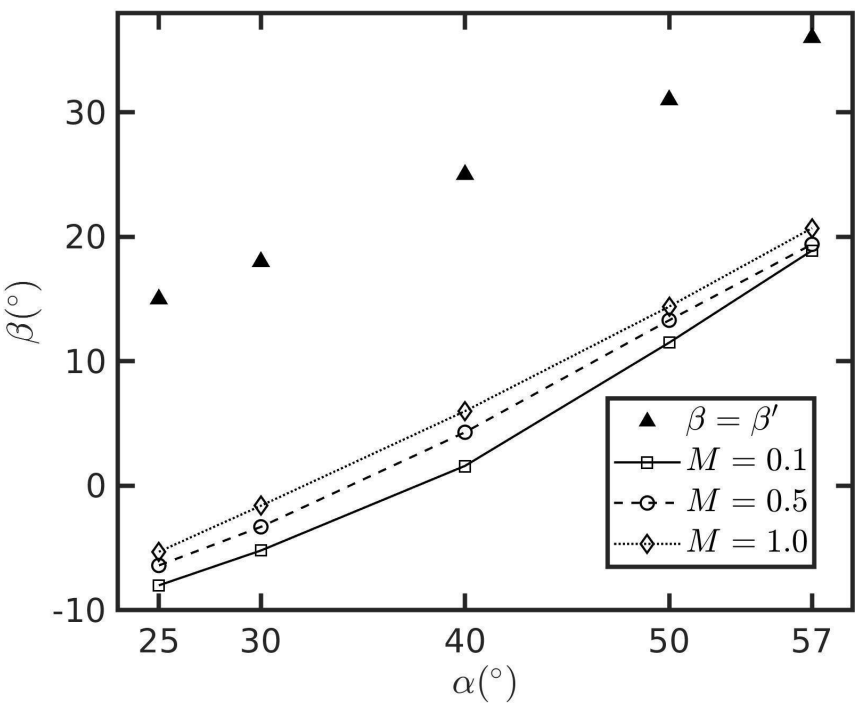

Fig. 9. $\beta$ vs $\alpha$ for $\mathrm{MgO}$ columnar structures according to the modified model, Eqn.9. The tilt angles obtained are smaller than (100) facet angle, i.e. $\beta<\beta^{\prime}$.

ing coefficient in Eqn. 9 is the main feature of our model that renders the tilting behavior of columnar structures abnormal.

\section{Film porosity}

For further analysis, we define some morphological parameters with the help of the cross section Fig. 8. As the columnar structures grow vertically, the column diameter $D$ increases until the side walls are completely shadowed, leading to column pinch-off. The thickness growth of the columnar structures can be defined as the growth stage in which the vertical dimension $h$ increases without the increase in $D$. The column pinch-off gives rise to a columnar void of size $\delta$. The columnar structures are neutrally stable with no true grain boundary formed. During the thickness growth, both $D$ and $\delta$ are maintained at constant values, and they are related by $D+\delta=d_{0}$, where $d_{0}$ is the initial seed spacing. Although the overall $\mathrm{MgO}$ columnar morphology is independent of the choice of $d_{0}$, the diameter $D$ reached by a columnar structure at thickness growth is determined by $D=d_{0}-\delta$. For this reason, $d_{0}$ is chosen to be the characteristic length scale for the simulations of thickness growth.

The porosity of the thin film originates from the formation of inter-columnar voids led by column pinch-off due to global shadowing. We define the void fraction $V_{f}$ of the thin film during thickness growth as $V_{f}=\delta /(D+\delta)=\delta / d_{0}$. The value of $\delta$ is dependent on the angle of global shadowing, also encapsulated by $\alpha$. Consequently, an $\alpha$ dependence of $V_{f}$ is expected. Further, the average growth direction makes an angle $\theta$ with the (100) top facet, and $\theta$ is related to $\beta$ by $\theta=90^{\circ}-\beta^{\prime}+\beta$. This angle can be used to determine a void fraction for idealised, fully faceted columnar structures $V_{f}^{\prime}$. Paritosh ${ }^{15}$ studied such void fraction and gave a formula for $V_{f}^{\prime}$ due to shadowing growth with no directional diffusion: 


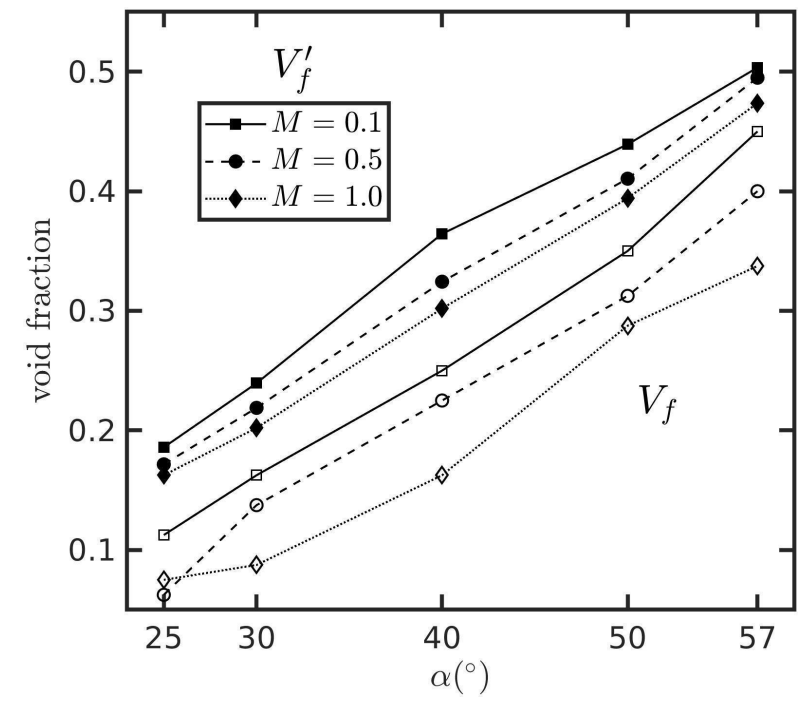

Fig. 10. The void fraction $V_{f}$ obtained simulations and the corresponding $V_{f}^{\prime}$ calculated by using Eqn. 10 .

$$
V_{f}^{\prime}=\frac{\cos (\beta-\theta) \sin (\alpha-\beta)}{\cos (\alpha) \sin (\theta)+\sin (\alpha-\beta) \sin (\beta)}
$$

Void fraction $V_{f}$ obtained by our simulations and $V_{f}^{\prime}$ according to Eqn. 10 are plotted in Fig. 10. Because $\theta$ varies with $M$, the corresponding $V_{f}^{\prime}$ also varies. It is evident that both $V_{f}$ and $V_{f}^{\prime}$ increase with an increasing deposition angle $\alpha$. The increase in porosity with increasing $\alpha$ has been commonly observed in obliquely deposited, biaxially textured thin films, e.g. $\mathrm{MgO}^{29}$, $\mathrm{Mg}^{11}$ and $\mathrm{CaF}_{2}{ }^{10}$. In addition to $\alpha$ dependence, faceting plays a role in determining the film porosity. Compared with $V_{f}^{\prime}$ which is evaluated in the no-faceting limit, $V_{f}$ takes on small values for the same $\alpha$. In all cases, measured $V_{f}$ from our simulations is less than $V_{f}^{\prime}$, showing that the effect of faceting realized by surface diffusion tends to reduce the density of the thin film. Correspondingly, the increase in adatom mobility reduces the void fraction, as evident in measured $V_{f}$, Fig. 10, for different $M$ values.

\section{E. Growth exponent}

Contrast to the void formation due to the pinch-off of columns in the in-flux direction, the columnar structures grow laterally in the $x^{\prime}$ direction and they impinge onto neighboring structures. The lateral dimension of a columnar structure marked by $W$ in Fig. 7 hence saturates after the event of impingement. Grain boundaries form between the columnar structures in the $x^{\prime} \mathrm{di}$ rection and the nature of which is determined by the orientation relationship. We investigate the scaling of $W$ and the vertical dimension $h$ before impingement occurs.

The vertical dimension for isolated nanocolumns is known to grow as a function of the vertical dimension during shadowing growth $^{45}$. For the anisotropic growth of multi-faceted $\mathrm{MgO}$

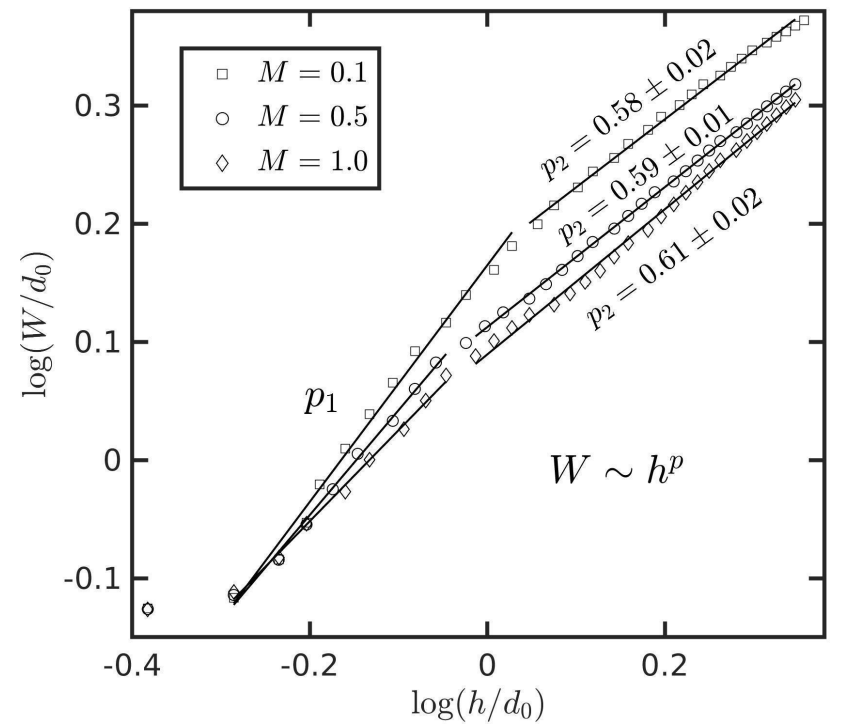

Fig. 11. Shadowing growth of multi-faceted $\mathrm{MgO}$ columnar structures $\left(\alpha=25^{\circ}\right)$ characterized by the spatial growth exponent $p$. The initial values of $W$ and $h$ are not included in the linear fitting of $p$.

columnar structures, Fig. 11, values of $W$ and $h$ during the shadowing growth with deposition angle $\alpha=25^{\circ}$ are plotted. A power law form $W \sim h^{p}$ can be fitted to the simulated shadowing growth with good precision. The spatial growth exponent $p$ is found to behave differently before and after the column pinchoff. The column pinch-off happens at approximately $h \approx d_{0}$, marked by the event that both side walls of a $\mathrm{MgO}$ column are shadowed. Before this event, the global shadowing is less effective because of the large spacing in between the columnar structures (for the initial separation $\delta=d_{0}-r_{0}$ at $h=W / 2$ ). The self-shadowing however is affected by the (100) facet angle $\beta^{\prime}$, which are predefined for the seeds according to different $\alpha$ values. A larger $\beta^{\prime}$ induces a larger shadowed area.

The initial growth exponent $p_{1}$ as shown in Fig. 12 as a result decreases with an increasing $\beta^{\prime}$ and equivalently $\alpha$. The adatom mobility is also found to influence the growth due to the fact that it controls the rate of formation of the (100) facet. After the column pinch-off, i.e. during thickness growth, the deposition flux reaches only the (100) top facets of the columnar structures and the growth is slowed down. The exponents at this growth stage amount to a mean $p_{2}=0.61 \pm 0.03$ and they are found to correlate less with $\alpha$. As compared with columns grown in the no-shadowing and no-faceting limits for which $p=1$, both the effects of shadowing and faceting tend to restrain the growth.

\section{F. $\mathrm{CaF}_{2}$ nanorods}

Here, we present a brief case study of the morphology and tilting behaviour of obliquely deposited, well-aligned $\mathrm{CaF}_{2}$ nanorods. They have important applications in preparing single-crystalline substrates ${ }^{46}$. The simulation methodology described in Sec. II D is followed. $\mathrm{CaF}_{2}$ nanorods have been 


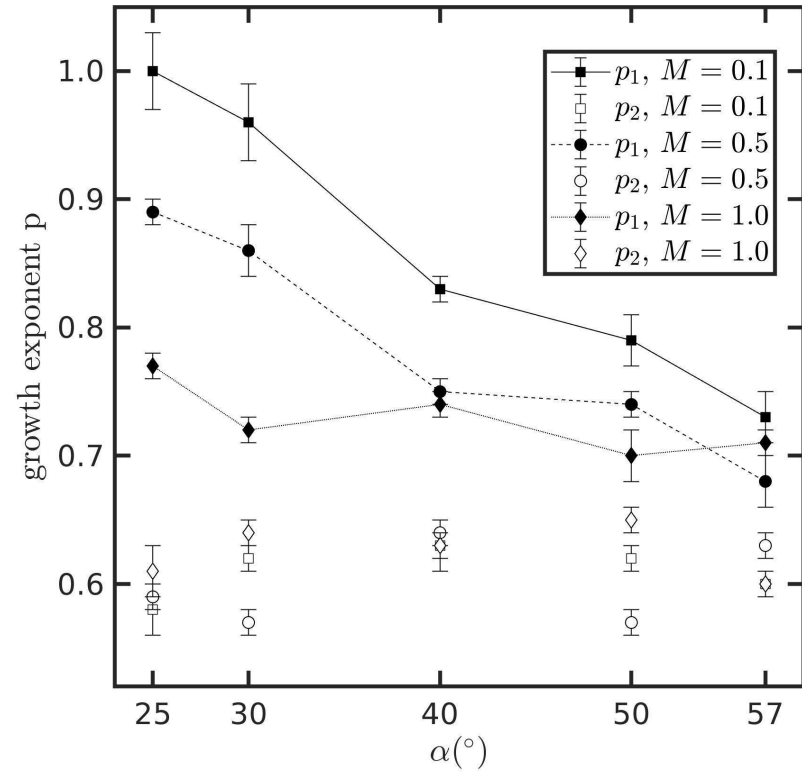

Fig. 12. The spatial growth exponents before and after the column pinch-off, $p_{1}$ and $p_{2}$ as functions of $\alpha$.

characterized to possess a $(111)\langle 121\rangle$ biaxial texture, with a variation in preferential orientation of the out-of-plane texture axis [111] for different $\alpha$ angles. Similar to $\mathrm{MgO}$ in Sec. II C, the orientation of seeds is predefined using the preferred [111] orientation values from experiments ${ }^{10}$. The surface energy density ${ }^{47} \gamma_{\{111\}}=0.465 \mathrm{Jm}^{-2}, \gamma_{\{110\}}=0.621 \mathrm{Jm}^{-2}$ and $\gamma_{\{100\}}=0.658 \mathrm{Jm}^{-2}$ calculated at $100^{\circ} \mathrm{C}$ are used for the construction of $\gamma(\boldsymbol{n})$, Eqn. 7, together with parameters $\omega=50$ and $a_{\{100\}}=0.1$. The $\gamma$-plot and the equilibrium shape reached in a long-time-scale evolution of a $\mathrm{CaF}_{2}$ crystal are shown in Fig. 13 (a). For the shadowing growth of columnar structures, we define a crystal coordinate system $x^{\prime}, y^{\prime}, z^{\prime}$, matching [ $\left.\begin{array}{lll}1 & \overline{1} & 0\end{array}\right],\left[\begin{array}{lll}1 & 1 & \overline{2}\end{array}\right]$ and [ $\left[\begin{array}{lll}1 & 1 & 1\end{array}\right]$ of $\mathrm{CaF}_{2}$ respectively.

Biaxially textured $\mathrm{CaF}_{2}$ thin films have been observed to exhibit a multi-faceted, roof-tile morphology ${ }^{10,18}$. In Fig. 13 (b) and (c), our simulation of the shadowing growth of a 1$\mathrm{D}$ array of $\mathrm{CaF}_{2}$ columnar structures aligned in the in-flux direction shows a multi-faceted columnar morphology. This morphology is consistent with experimentally observed $\mathrm{CaF}_{2}$ nanorods. Comparing to $\mathrm{MgO},\{111\}$-type facets of $\mathrm{CaF}_{2}$ are known to have the lowest surface energy. The terminating facets on $\mathrm{CaF}_{2}$ nanorods are of $\{111\}$ type as indicated by the color of the facets in Fig. 13. The fan angle of the (11) $)$ "roof-tile" facet is equal to $70^{\circ}$, which corresponds to one of the trihedral angles formed by $(11 \overline{1}),(1 \overline{1} 1)$ and $(\overline{1} 11)$. This differs from the $90^{\circ}$ angle of the (100) top facet of $\mathrm{MgO}$ (Fig. 7), yielding a narrower fan-like shape.

Well-isolated nanorods were previously fabricated at large deposition angles $\alpha^{10,18}$. The separations between nanorods facilitated the imaging of the individual single-crystal columnar structures, allowing the average tilt angles $\beta$ to be measured quantitatively and accurately for various $\alpha$ angles using microscopy. Systematic $\beta$ vs $\alpha$ measurement is available, which has not so far been obtained for $\mathrm{MgO}$ due to its highly

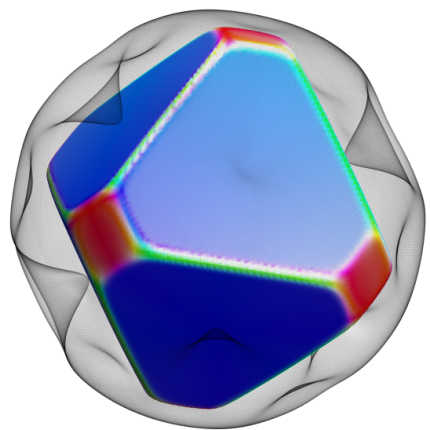

(a)

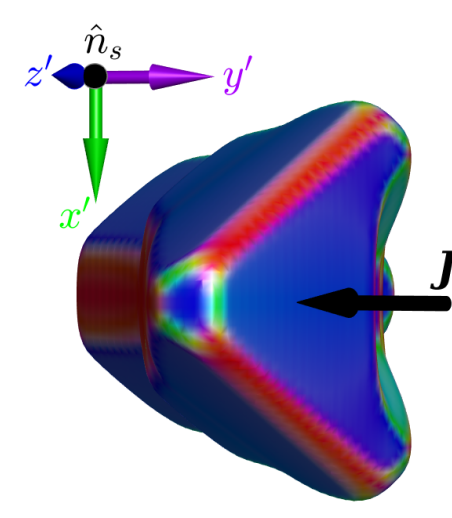

(b)

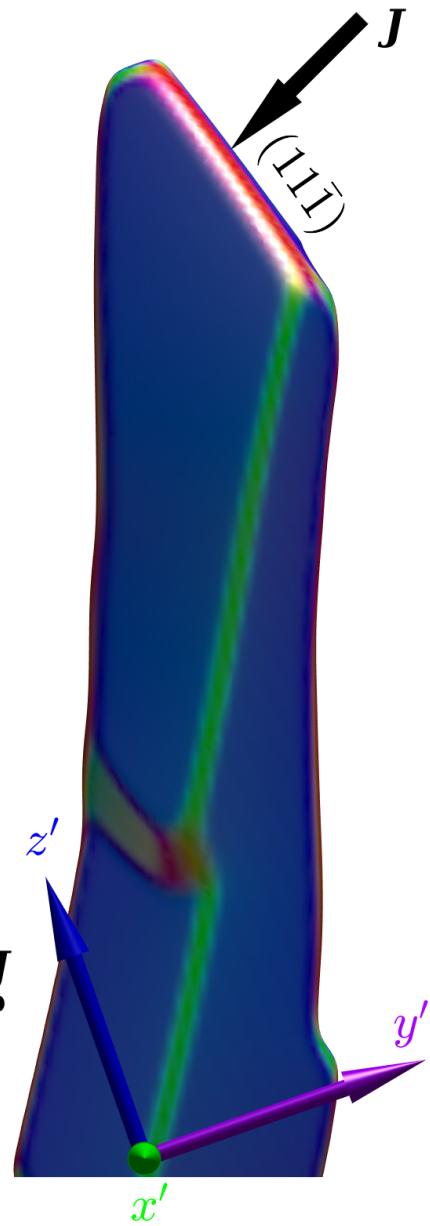

(c)
Fig. 13. (a) $\gamma$-plot and equilibrium shape of a $\mathrm{CaF}_{2}$ crystal. The top view (b), and the side view (c) of simulated multi-faceted $\mathrm{CaF}_{2}$ columnar morphology, with only one of the 1-D array of isolated structures shown. It is terminated by predominantly $\{111\}$ facets.

anisotropic morphology. In Fig. 14, results obtained by the simulations are compared with reported values for $\mathrm{CaF}_{2}$ at $100{ }^{\circ} \mathrm{C}^{10}$. The abnormal tilting behavior is reproduced by our model. The large deviation from any known existing predictions is attributed to faceting, similar to $\mathrm{MgO}$. Yet the increase in adatom mobility results in a decrease in $\beta$, as opposed to an increase in $\beta$ for $\mathrm{MgO}$ (Fig. 9). This suggests that the effect of faceting on the tilting behavior of columnar structures is material-specific.

\section{CONCLUSIONS}

In conclusion, a material-specific continuum model has been proposed to study the detailed morphological evolution of obliquely deposited thin films. The proposed model explicitly accounts for the finite adatom mobility, material-specific surface energies and sticking coefficient proportional to the com- 


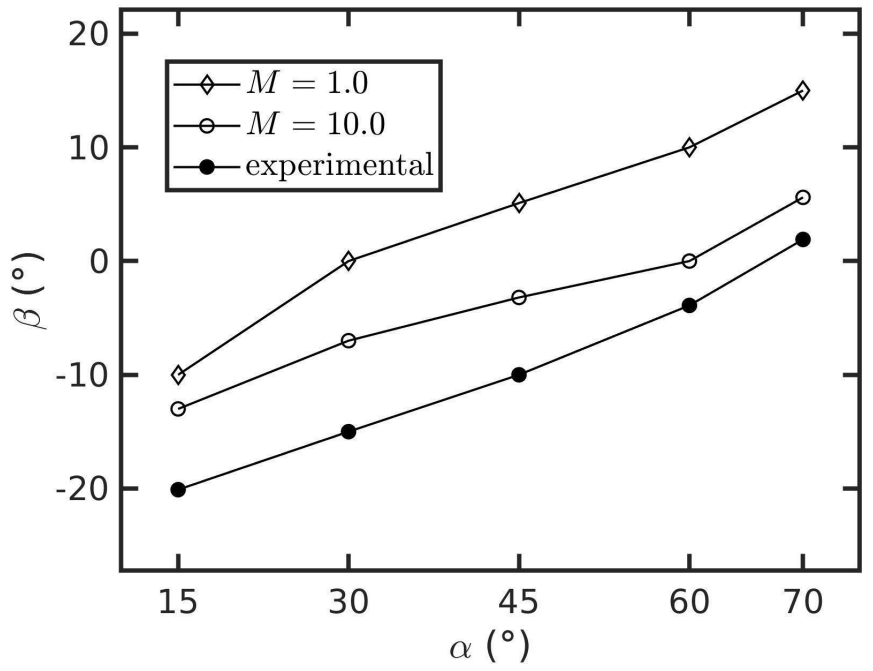

Fig. 14. $\beta$ vs $\alpha$ of simulated $\mathrm{CaF}_{2}$ nanorods $\left(\alpha=45^{\circ}\right)$ compared with experimental results ${ }^{10}$. The tilting behavior is controlled by adatom mobility, the magnitude of which is represented in the model by coefficient $M$.

ponent of the adatom mobility parallel to the surface. A wellknown MgO-OAD system is used to demonstrate the model and the most significant achievements are as follow:

i The proposed flux-dependent sticking coefficient is responsible for the development of abnormal tilting.

ii Experimentally observed roof-tile, multi-faceted columnar morphologies characteristic of $\mathrm{MgO}$ nanocolumns are reproduced by means of numerical simulations.

iii The shadowing growth of $\mathrm{MgO}$ columnar structures according to our model demonstrates column bundling, commonly observed in biaxially textured thin films. It is attributed to global shadowing by our analysis.

iv The role of faceting is manifested in the development of multi-faceted morphology, abnormal tilting, film porosity and the growth of lateral dimension of $\mathrm{MgO}$.

$\mathrm{v} \alpha$ dependence of $\beta$ and the void fraction is found and the relationships are consistent with experiments.

vi Both tilting and film porosity are found to be controlled by adatom mobility.

The morphological parameters studied here have important implications in the applications of e.g. single-crystalline substrates and coated conductor templates. Our proposed model can be applied to other nano-structured systems in general of practical interest. This is verified and supported by the implementation of a $\mathrm{CaF}_{2}-\mathrm{OAD}$ system.

\section{ACKNOWLEDGMENTS}

This work has been supported in part by New Zealand Ministry for Business Innovation and Employment (MBIE) grant

\section{No. CONT-42986-HVM.}

\section{DATA AVAILABILITY}

The data that support the findings of this study are available from the corresponding author upon reasonable request

\section{REFERENCES}

${ }^{1}$ Z. Aabdin, M. Dürrschnabel, M. Bauer, R. Semerad, V. Große, W. Prusseit, and O. Eibl, "Growth behavior of $\mathrm{DyBa}_{2} \mathrm{Cu}_{3} \mathrm{O}_{7}-\delta$ thin films deposited by inclined substrate deposition for coated conductors," Physics Procedia 36 1445 - 1449 (2012).

${ }^{2}$ F. Tang, T. Karabacak, P. Morrow, C. Gaire, G.-C. Wang, and T.-M. Lu, "Texture evolution during shadowing growth of isolated Ru columns," Phys Rev. B 72, 165402 (2005).

${ }^{3}$ M. Hilse, Y. Takagaki, J. Herfort, M. Ramsteiner, C. Herrmann, S. Breuer, L. Geelhaar, and H. Riechert, "Ferromagnet-semiconductor nanowire coaxial heterostructures grown by molecular-beam epitaxy," Appl. Phys. Lett. 95, 133126 (2009).

${ }^{4}$ S. P. Stagon and H. Huang, "Controllable growth of aluminum nanorods using physical vapor deposition,” Nanoscale Res Lett 9, 400 (2014).

${ }^{5}$ M. Pelliccione and T.-M. Lu, Evolution of Thin Film Morphology (SpringerVerlag, 2004).

${ }^{6}$ G.-C. Wang and T.-M. Lu, RHEED Transmission Mode and Pole Figures (Springer New York, New York, 2014).

${ }^{7}$ H. H. J. M. Nieuwenhuizen, "Microfractography of thin films," Philips Tech. Rev. , 87-91 (1966).

${ }^{8}$ R. Tait, T. Smy, and M. Brett, "Modelling and characterization of columnar growth in evaporated films," Thin Solid Films 226, 196-201 (1993).

${ }^{9}$ H. Zhu, W. Cao, G. K. Larsen, R. Toole, and Y. Zhao, "Tilting angle of nanocolumnar films fabricated by oblique angle deposition,” J. of Vacuum Science \& Technology B 30, 030606 (2012).

${ }^{10}$ C. Gaire, P. Snow, T.-L. Chan, W. Yuan, M. Riley, Y. Liu, S. B. Zhang, G.-C. Wang, and T.-M. Lu, "Morphology and texture evolution of nanostructured CaF2films on amorphous substrates under oblique incidence flux," Nanotechnology 21, 445701 (2010).

${ }^{11}$ F. Tang, T. Parker, H.-F. Li, G.-C. Wang, and T.-M. Lu, "Unusual magnesium crystalline nanoblades grown by oblique angle vapor deposition," J. Nanosci Nanotech. 7, 3239-3244 (2007).

${ }^{12}$ P. Ghekiere, S. Mahieu, G. De Winter, R. De Gryse, and D. Depla, "Scanning electron microscopy study of the growth mechanism of biaxially aligned magnesium oxide layers grown by unbalanced magnetron sputtering," Thin Solid Films 493, 129-134 (2005).

${ }^{13} \mathrm{~S}$. Lichter and J. Chen, "Model for columnar microstructure of thin solid films," Phys. Rev. Lett. 56, 1396-1399 (1986).

${ }^{14}$ P. Keblinski, A. Maritan, F. Toigo, R. Messier, and J. R. Banavar, "Continuum model for the growth of interfaces," Phys. Rev. E 53, 759-778 (1996).

${ }^{15}$ Paritosh and D. Srolovitz, "Shadowing effects on the microstructure of obliquely deposited films," J. Appl. Phys. 91, 1963-1972 (2002).

${ }^{16}$ M. Dürrschnabel, Z. Aabdin, V. Große, M. Bauer, G. Sigl, W. Prusseit, and $\mathrm{O}$. Eibl, "Growth of biaxially-textured $\mathrm{MgO}$ buffer layers by inclined substrate deposition," Physics Procedia 36, 1546-1551 (2012).

${ }^{17}$ M. Bauer, R. Metzger, R. Semerad, P. Berberich, and H. Kinder, "Inclined substrate deposition by evaporation of magnesium oxide for coated conductors," MRS Proc. 585, 35 (1999).

${ }^{18}$ H.-F. Li, T. Parker, F. Tang, G.-C. Wang, T.-M. Lu, and S. Lee, "Biaxially oriented CaF2 films on amorphous substrates," J. Cryst. Growth 310, 36103614 (2008).

${ }^{19}$ F. Tang, G.-C. Wang, and T.-M. Lu, "In situ reflection high energy electron diffraction surface pole figure study of biaxial texture evolution in anisotropic mg nanoblades during shadowing growth,” J. Appl. Phys. 102 014306 (2007).

${ }^{20}$ R. Deng, P. Muralt, and D. Gall, "Biaxial texture development in aluminum nitride layers during off-axis sputter deposition," J. of Vacuum Science \& Technology A 30, 051501 (2012). 
${ }^{21}$ M. C. Tai, A. R. Gentle, M. D. Arnold, and M. B. Cortie, "Spontaneous growth of polarizing refractory metal 'nano-fins,", Nanotechnology 29 , 105702 (2018)

${ }^{22}$ L. Abelmann and C. Lodder, "Oblique evaporation and surface diffusion," Thin Solid Films 305, 1-21 (1997).

${ }^{23}$ M. Pelliccione, T. Karabacak, C. Gaire, G.-C. Wang, and T.-M. Lu, "Mound formation in surface growth under shadowing," Phys. Rev. B 74, 125420 (2006).

${ }^{24}$ M. Pelliccione and T.-M. Lu, "Non-local effects in thin film growth," Mod. Phys. Lett. B 21, 1207-1225 (2007).

${ }^{25}$ S. Mahieu, P. Ghekiere, D. Depla, and R. De Gryse, "Biaxial alignment in sputter deposited thin films," Thin Solid Films 515, 1229-1249 (2006).

${ }^{26}$ F. Chen and J. Shen, "Efficient energy stable schemes with spectral discretization in space for anisotropic cahn-Hilliard systems," Commun. comput. phys. 13, 1189-1208 (2013)

${ }^{27}$ F. Chen and J. Shen, "Efficient spectral-galerkin methods for systems of coupled second-order equations and their applications," J. Comput. Phys. 231, 5016-5028 (2012)

${ }^{28}$ P. Ghekiere, S. Mahieu, R. De Gryse, and D. Depla, "Structure evolution of the biaxial alignment in sputter-deposited $\mathrm{MgO}$ and Cr," Thin Solid Films 515, 485-488 (2006)

${ }^{29}$ Y. Xu, C. H. Lei, B. Ma, H. Evans, H. Efstathiadis, R. Manisha, M. Massey, U. Balachandran, and R. Bhattacharya, "Growth of textured $\mathrm{MgO}$ through ebeam evaporation and inclined substrate deposition," Supercond. Sci. Technol. 19, 835-843 (2006)

${ }^{30} \mathrm{~S}$. Torabi and J. Lowengrub, "Simulating interfacial anisotropy in thin-film growth using an extended cahn-Hilliard model," Phys. Rev. E 85, 041603 (2012).

${ }^{31}$ M. Salvalaglio, R. Backofen, R. Bergamaschini, F. Montalenti, and A. Voigt, "Faceting of equilibrium and metastable nanostructures: A phase-field model of surface diffusion tackling realistic shapes," Cryst. Growth Des. 15, 27872794 (2015)

${ }^{32}$ M. Salvalaglio, R. Backofen, A. Voigt, and F. Montalenti, "Morphological evolution of pit-patterned si(001) substrates driven by surface-energy reduction," Nanoscale Res Lett 12 (2017), 10.1186/s11671-017-2320-5.

${ }^{33}$ M. Salvalaglio, R. Bergamaschini, R. Backofen, A. Voigt, F. Montalenti, and L. Miglio, "Phase-field simulations of faceted Ge/Si-crystal arrays, merging into a suspended film,” Appl. Surf. Sci. 391, 33-38 (2017).

${ }^{34}$ E. J. Siem and W. C. Carter, "Orientation-dependent surface tension functions for surface energy minimizing calculations," J Mater Sci 40, 3107-3113 (2005)

${ }^{35}$ A. Sternig, S. Klacar, J. Bernardi, M. Stöger-Pollach, H. Grönbeck, and O. Diwald, "Phase separation at the nanoscale: Structural properties of $\mathrm{BaO}$ segregates on MgO-based nanoparticles," J. Phys. Chem. C 115, 1585315861 (2011).

${ }^{36} \mathrm{C}$. Herring, "Some theorems on the free energies of crystal surfaces," Phys. Rev. 82, 87-93 (1951)

${ }^{37}$ K. Hasegawa, Y. Nakamura, T. Izumi, and Y. Shiohara, "Investigation of texture development on $\mathrm{MgO}$ films prepared by inclined substrate deposition with electron-beam evaporation,” Physica C 357-360, 967-970 (2001).

${ }^{38}$ B. Ma, M. Li, Y. Jee, R. Koritala, B. Fisher, and U. Balachandran, "Inclinedsubstrate deposition of biaxially textured magnesium oxide thin films for YBCO coated conductors," Physica C 366, 270-276 (2002).

${ }^{39}$ K. Hasegawa, Y. Nakamura, T. Izumi, and Y. Shiohara, "Comparative study on texture development of $\mathrm{MgO}$ and YSZ films grown by inclined substrate deposition technique," Physica C 378-381, 955-959 (2002).

${ }^{40}$ A. van der Drift, "Evolutionary selection, a principle governing growth orientation in vapour-deposited layers," Philips Res. Rep. 22, 267-288 (1967).

${ }^{41}$ M. Salvalaglio, R. Backofen, and A. Voigt, "Thin-film growth dynamics with shadowing effects by a phase-field approach,” Phys. Rev. B 94, 235432 (2016).

${ }^{42}$ D. L. Smith, Thin-film deposition: principles and practice (McGraw-Hill, New York, 1995)

${ }^{43}$ T. Michely and J. Krug, Islands, Mounds and Atoms (Springer-Verlag Berlin Heidelberg, 2004).

${ }^{44}$ D. P. Woodruff, "How does your crystal grow? a commentary on burton, cabrera and frank (1951) 'the growth of crystals and the equilibrium structure of their surfaces'," Philosophical transactions. Series A, Mathematical, physical, and engineering sciences 373, 20140230 (2015).
${ }^{45}$ T. Karabacak, J. P. Singh, Y.-P. Zhao, G.-C. Wang, and T.-M. Lu, "Scaling during shadowing growth of isolated nanocolumns," Phys. Rev. B 68, 125408 (2003)

${ }^{46}$ C. Gaire, P. Clemmer, H.-F. Li, T. Parker, P. Snow, I. Bhat, S. Lee, G.-C Wang, and T.-M. Lu, "Small angle grain boundary Ge films on biaxial CaF2/glass substrate,” J. Cryst. Growth 312, 607-610 (2010).

${ }^{47} \mathrm{~S}$. Bebelis and P. Nikolopoulos, "Temperature dependence of the surface energy of the low-index planes of $\mathrm{CaF} 2, \mathrm{BaF} 2$ and $\mathrm{SrF} 2$," J. of Materi Eng and Perform 26, 1223-1228 (2017). 


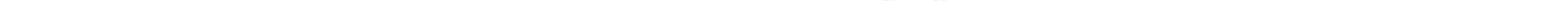




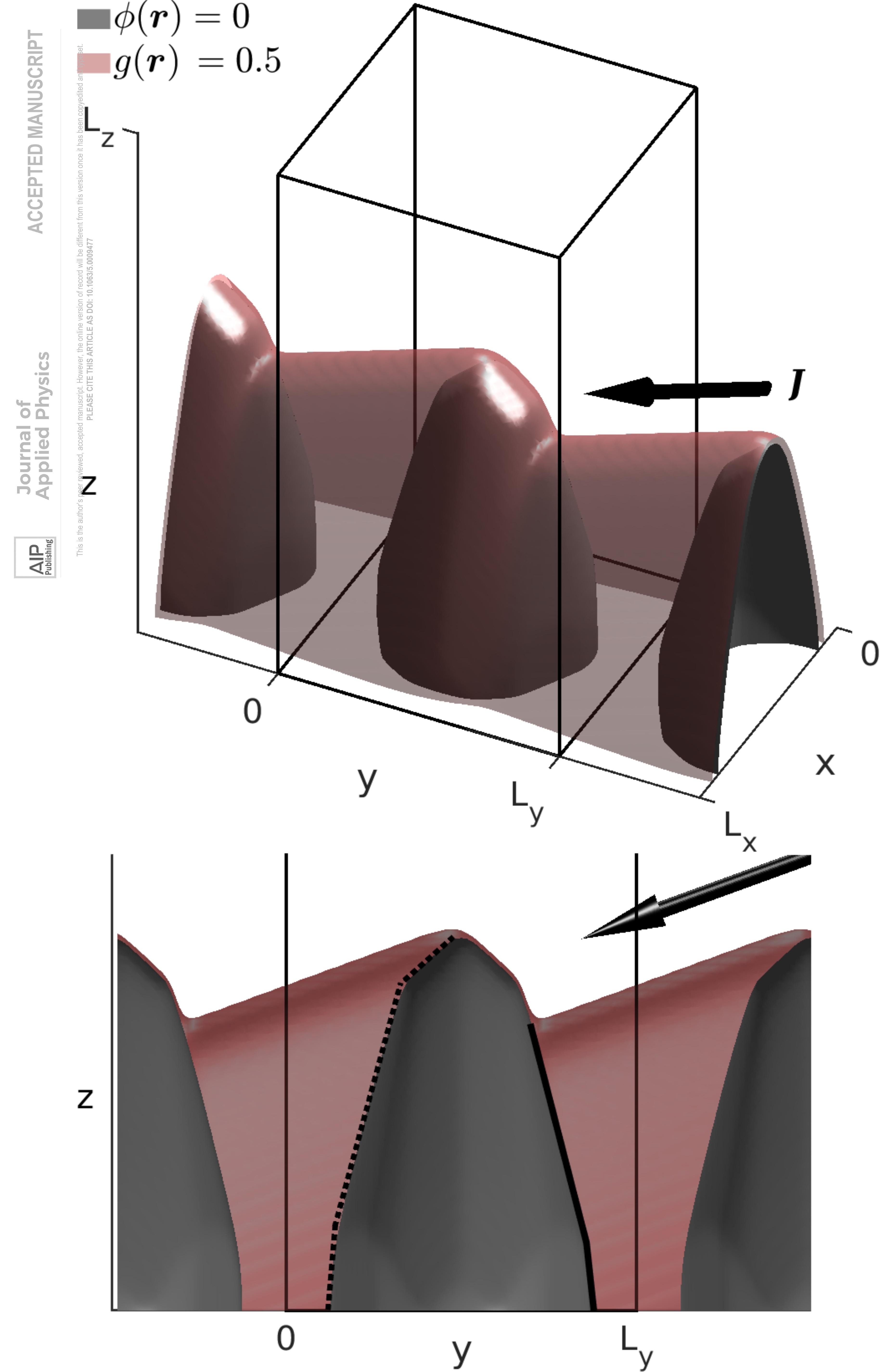




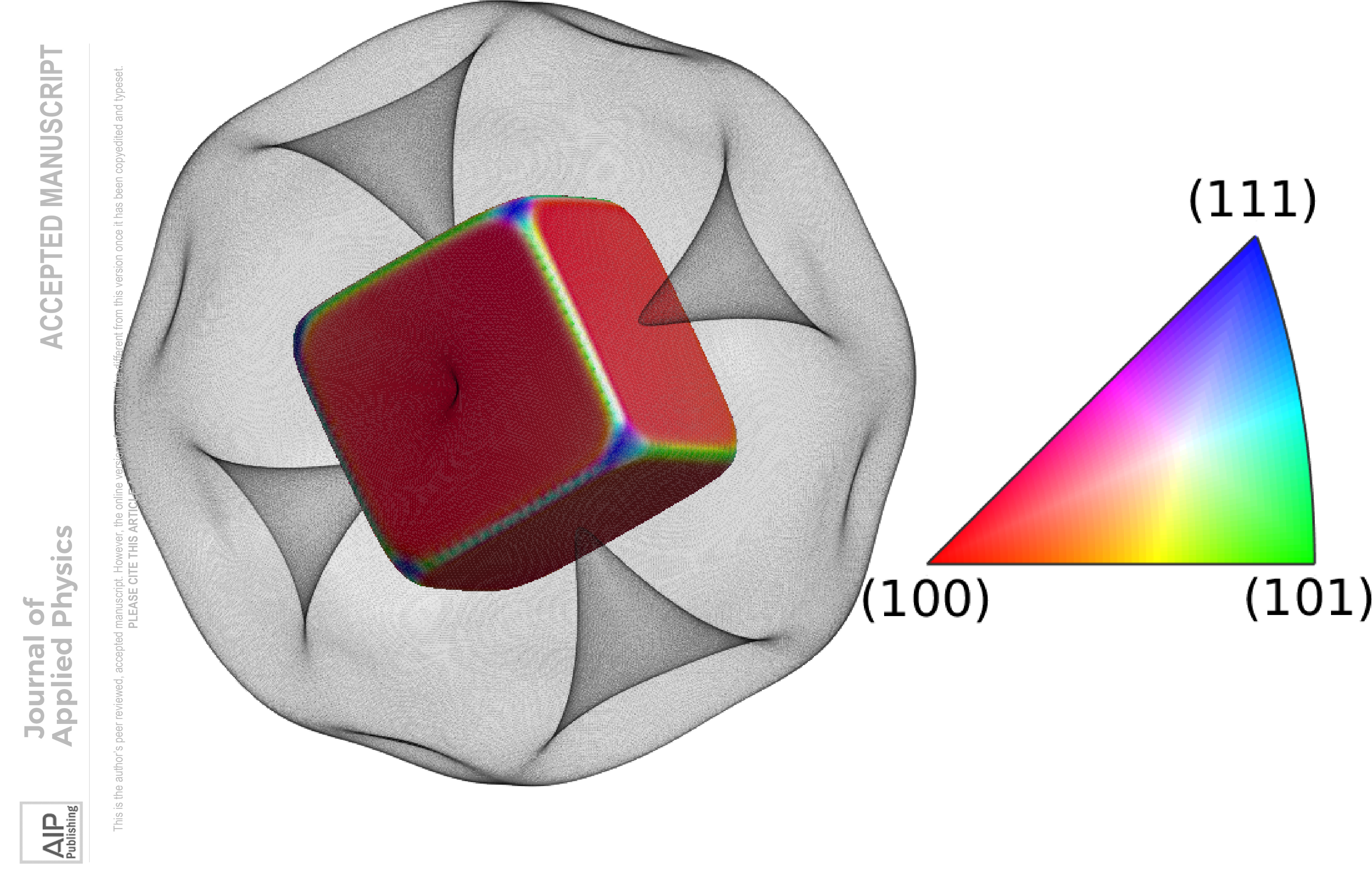




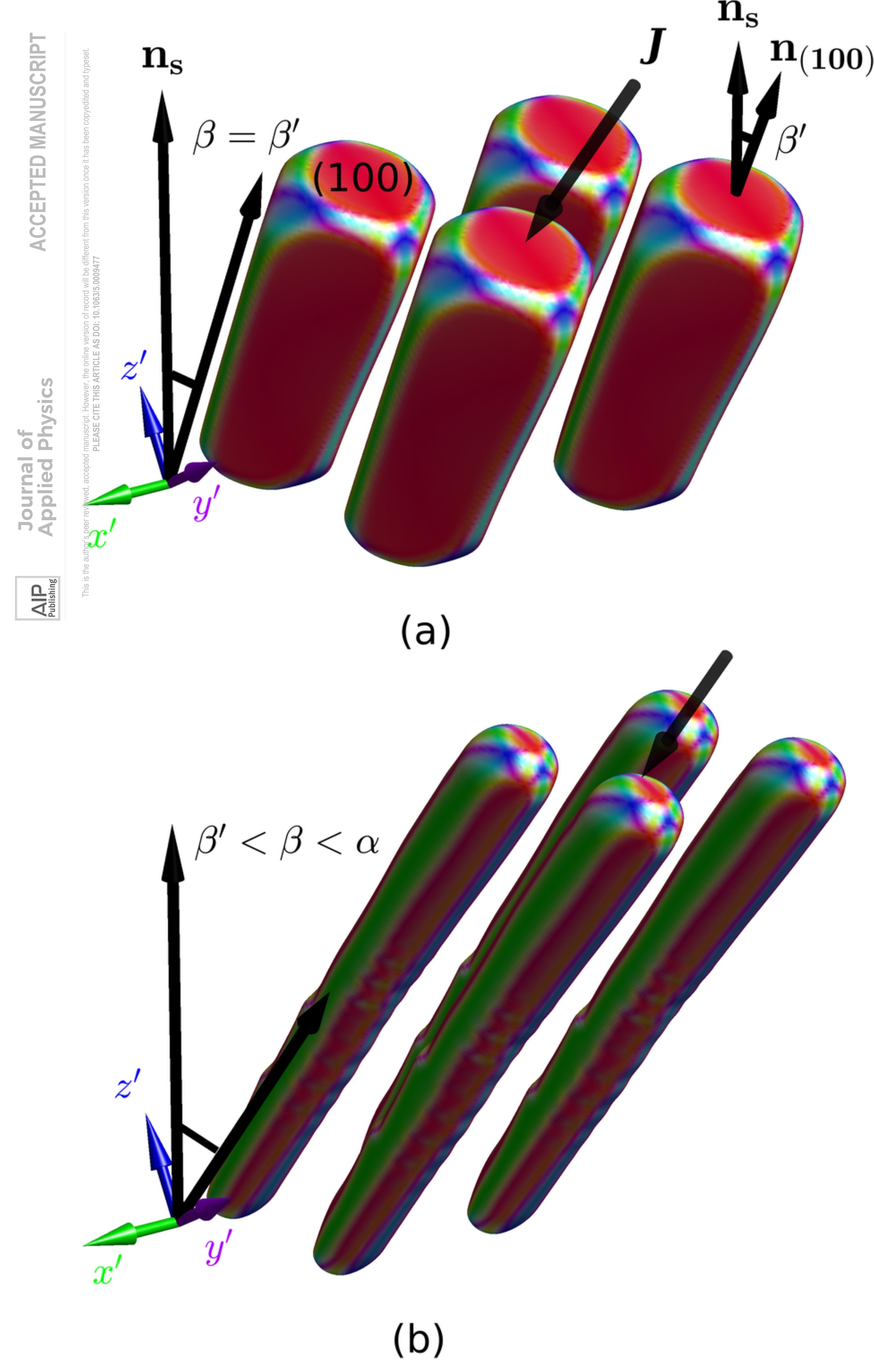




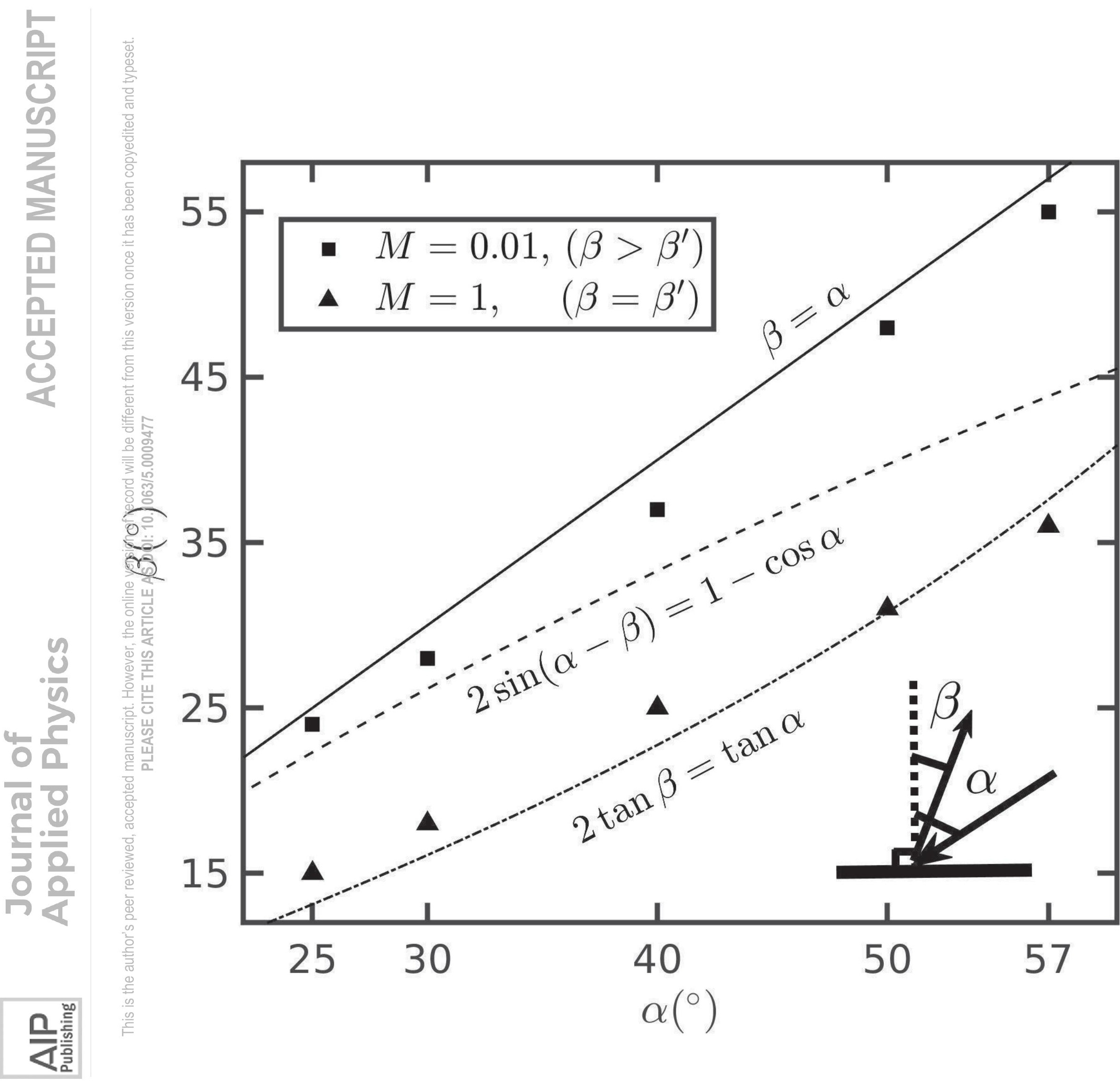









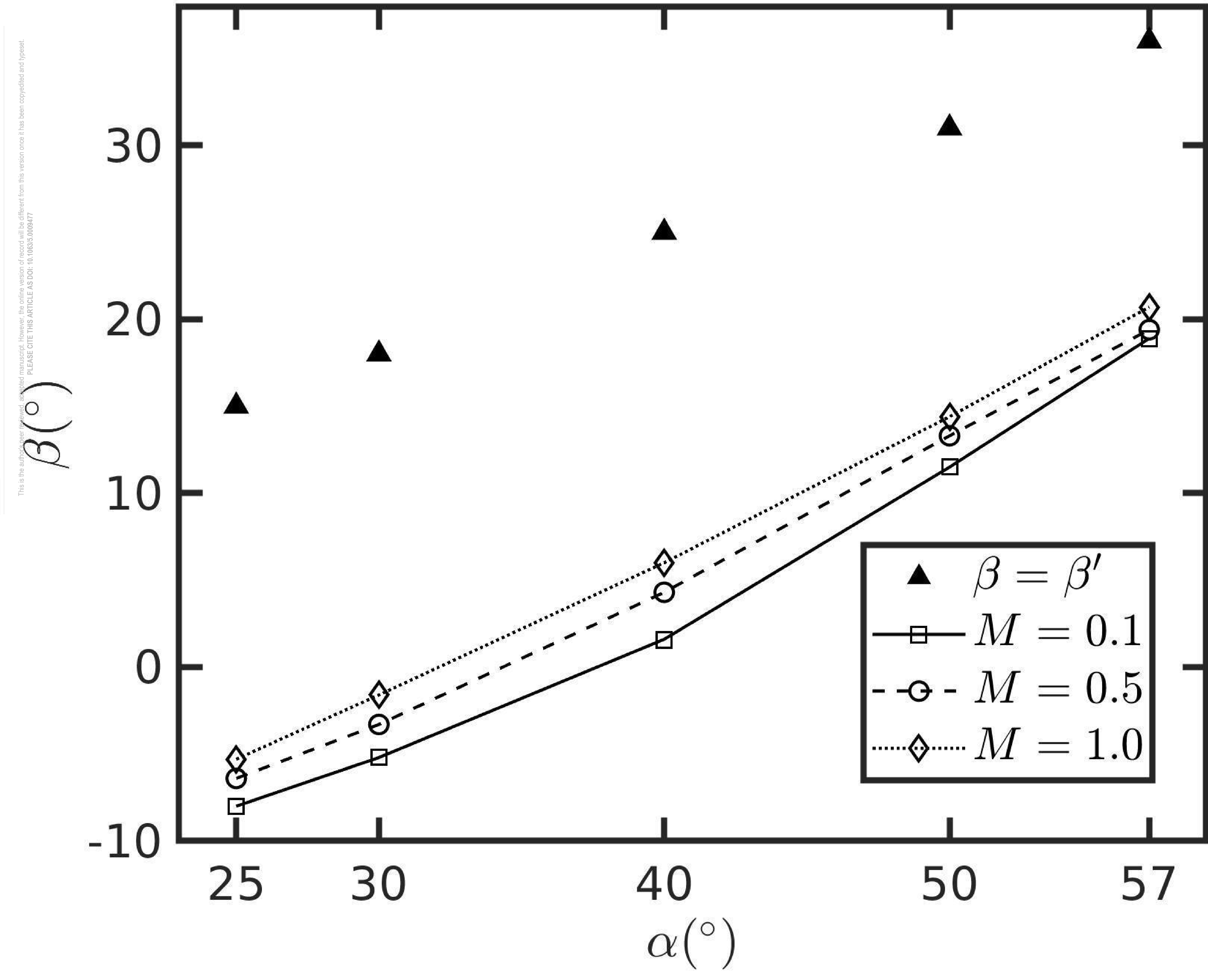




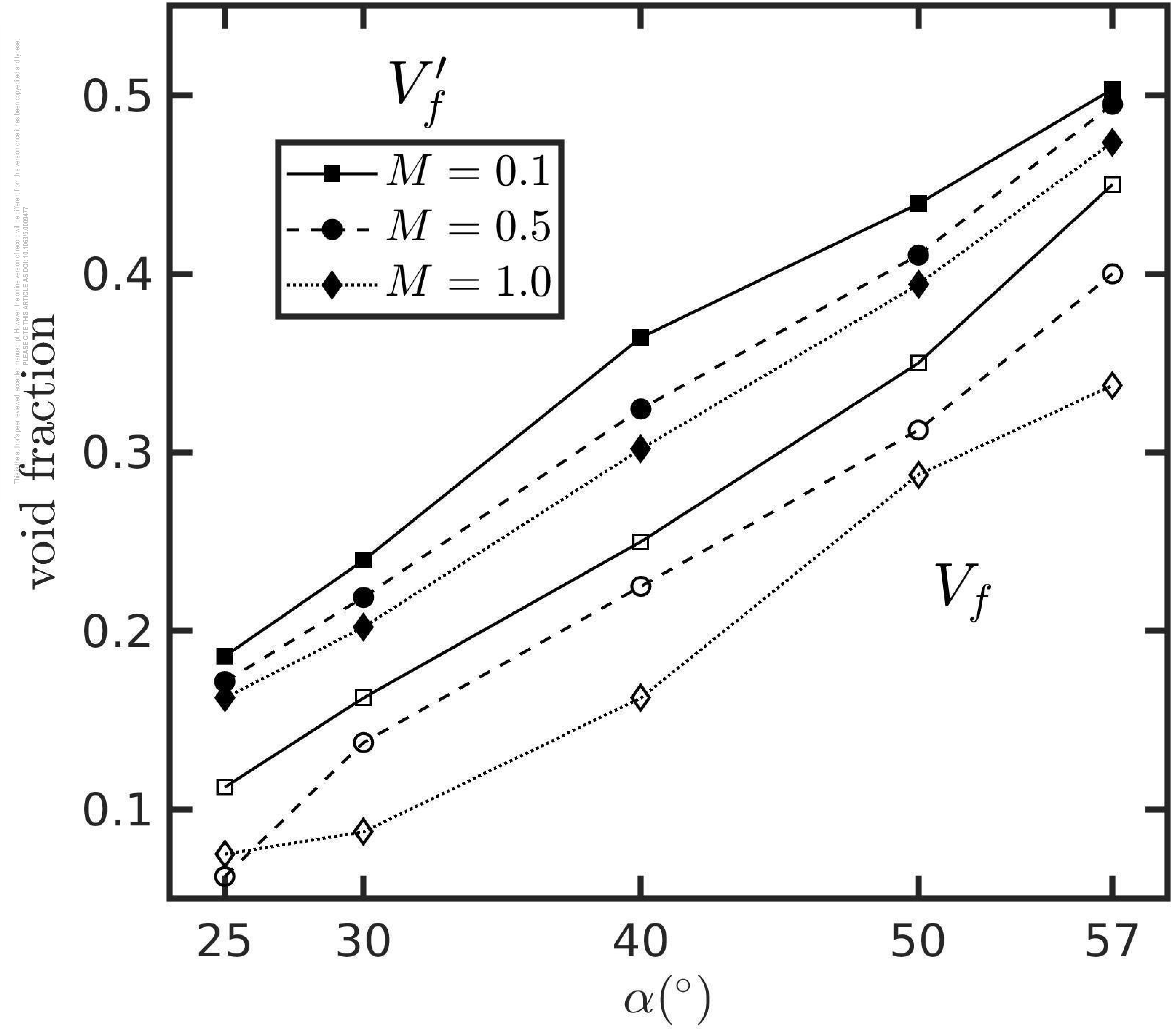




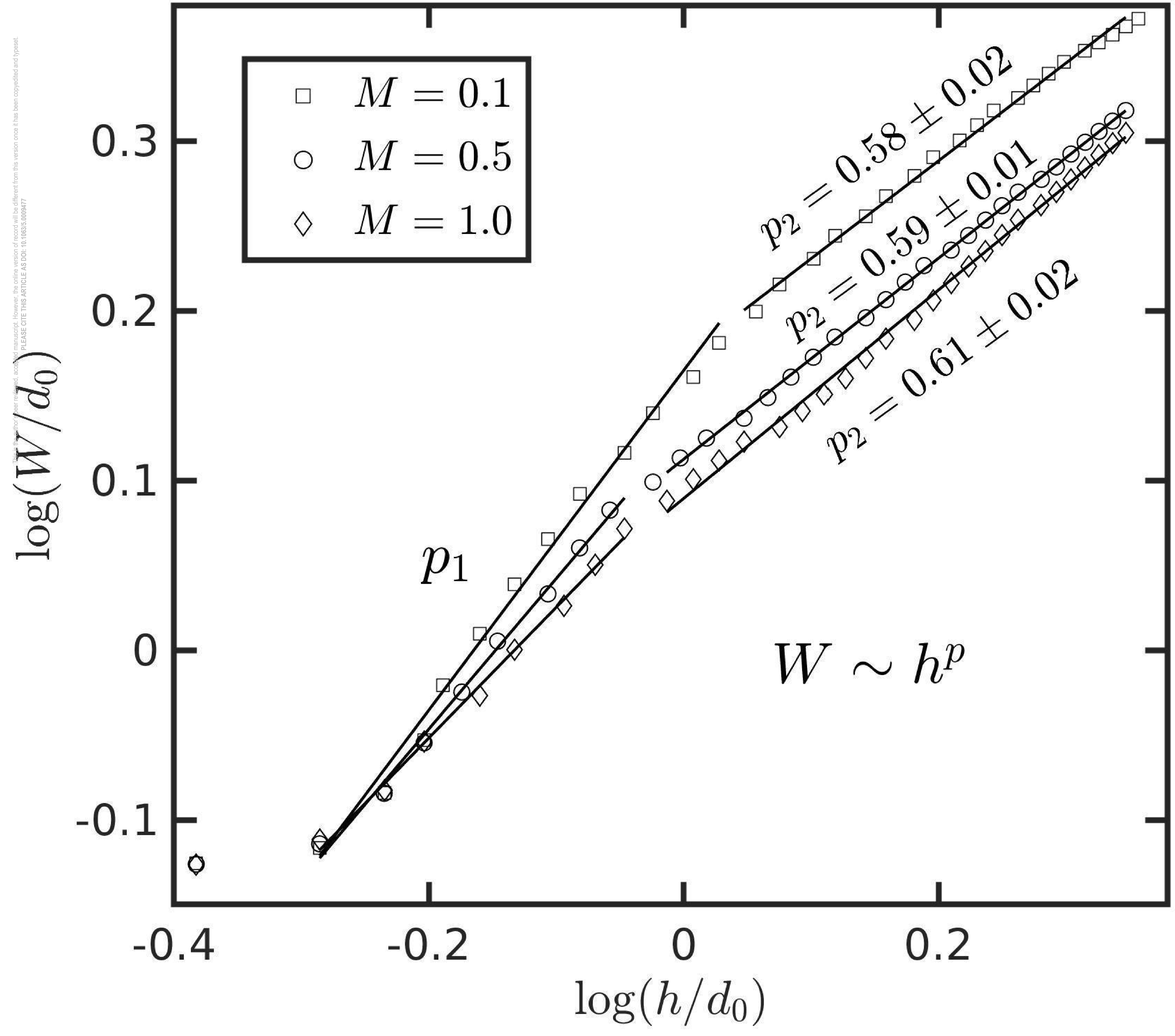




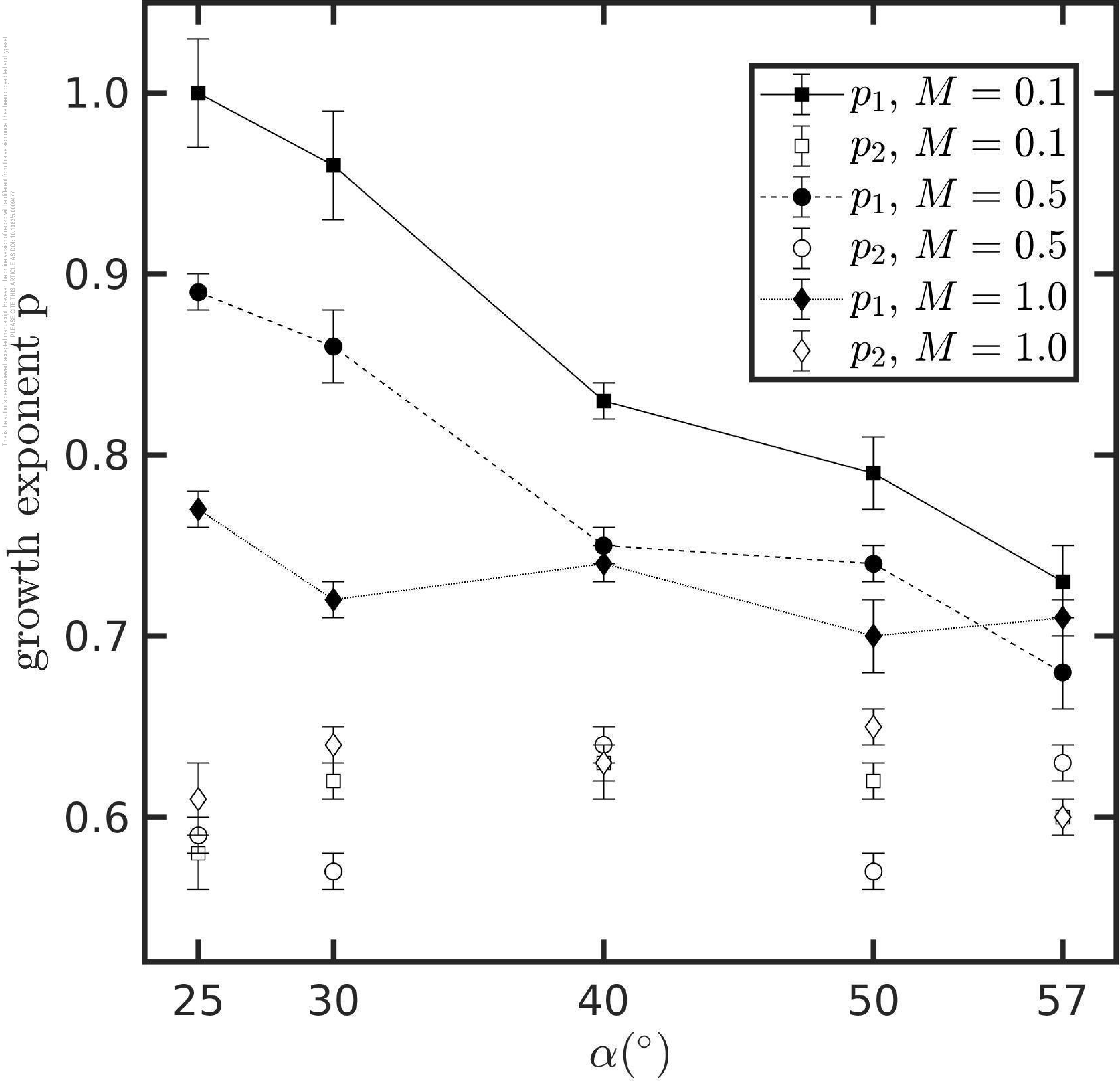


